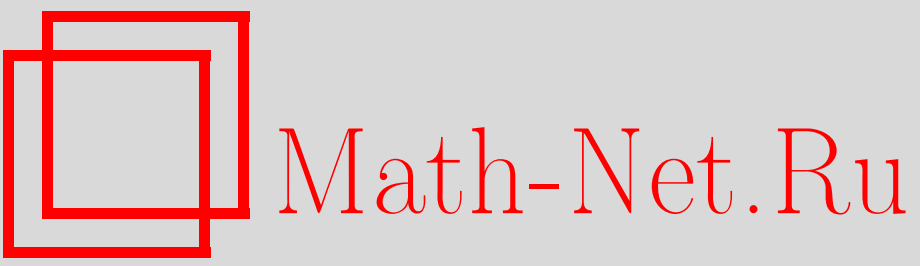

И. А. Дынников, Алгоритмы распознавания в теории узлов, УМН, 2003, том 58, выпуск 6, 45-92

DOI: https://doi.org/10.4213/rm675

Использование Общероссийского математического портала Math-Net.Ru подразумевает, что вы прочитали и согласны с пользовательским соглашением

http://www.mathnet.ru/rus/agreement

Параметры загрузки:

IP : 54.166 .219 .16

26 апреля 2023 г., 03:58:53 


\title{
АЛГОРИТМЫ РАСПОЗНАВАНИЯ В ТЕОРИИ УЗЛОВ
}

\author{
И. А. Дынников
}

\begin{abstract}
В работе обсуждаются вопросы, связанные с проблемой построения алгоритмов для сравнения узлов и зацеплений. Дается обзор существующих подходов и основных резулштатов в этой области. В частности, обсуждаются различные комбинаторные способы представления зацеплений, излагаются алгоритм Хакена распознавания тривиального узла и схема построения общего алгоритма сравнения зацеплений, основанного на идеях Хакена; описывается подход, основанньй на представлении зацеплений замкнутыми косами; для групп кос описываются известные алгоритмы для решения проблемш равенства и проблемш сопряженности; обсуждается сложность рассматриваемых алгоритмов. В работе приводится также новый способ комбинаторного описания узлов и основанный на нем новый алгоритм распознавания тривиалного узла, использующий процедуру монотонного упрощения. В завершение работы сформулировано несколько задач, решение которых позволило бы продвинуться в "алгоритмизации" теории узлов.

Библиография: 76 названий.
\end{abstract}

\section{СОДЕРЖАНИЕ}

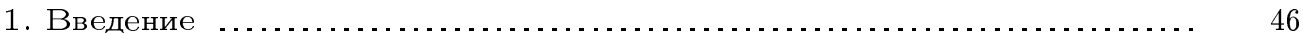

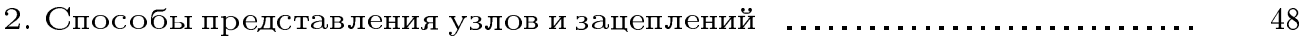

2.1. Выбор комбинаторной модели .............................. 48

2.2. Плоские диаграммы … . . . . . . . . . . . . . . . . . . . . . . . . . . . . . 49

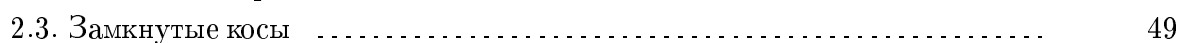

2.4. Прямоугольные диаграммы ................................ 51

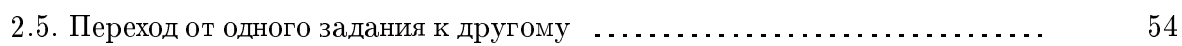

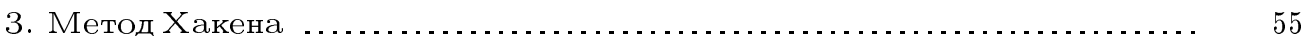

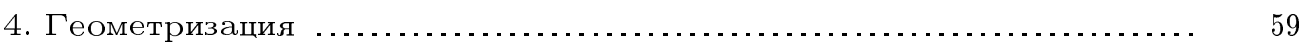

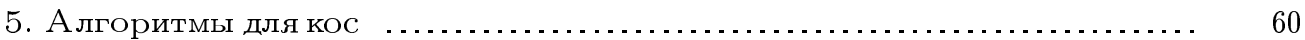

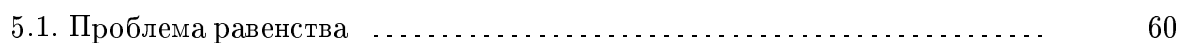

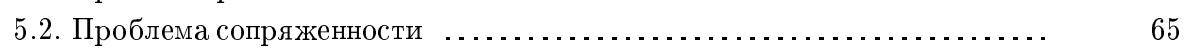

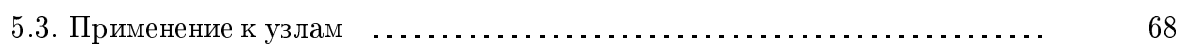

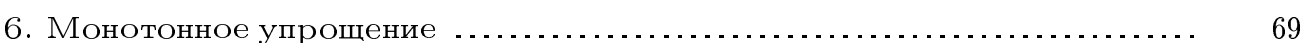

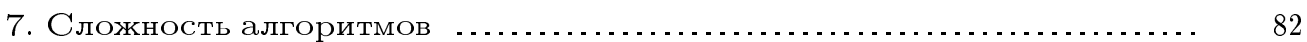

8. Некоторые новые алгоритмические задачи теории узлов ........... 84 
8.1. Флайпы прямоугольных диаграмм ... . . . . . . . . . . . . . . . . . . . . . . .

8.2. Алгоритм Хакена и каноническая триангуляция ...................

8.3. Сведение классификации зацеплений к проблеме равенства в конечно определенной полугруппе ................................. 87

8.4. Вложение группы кос в конечно определенные группы ............. 88

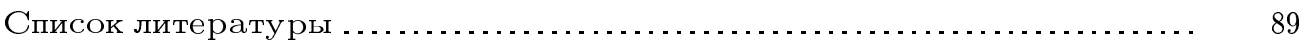

\section{1. Введение}

Вопрос о возможности сравнения двух данных узлов в $\mathbb{R}^{3}$ интересует математиков по меньшей мере с конца XIX века. Первые таблищы узлов малой сложности появились уже в [1], [2]. Основы математической теории узлов сформировались в начале ХХ века в работах Дж. Александера, М. Дэна, Г. Зейферта и К. Райдемайстера. Тогда же появились первые доказательства нетривиальности конкретных узлов.

В работах [3], [4] узлы были представлены в чисто комбинаторных терминах - с помощью регулярных проекций и движений Райдемайстера. Позднее было придумано другое комбинаторное описание - с помошњю замкнутых кос и движений Маркова [5], [6]. Сами группы кос были определены Э. Артином [7], [8], который, кроме того, решил для них проблему равенства. В первой половине XX века появился ряд доказательств нетривиальности и неэквивалентности конкретных узлов (например, с использованием многочлена Александера), однако до середины 1950-х годов не было придумано ни одного обшего алгоритма, устанавливающего, является ли данный узел тривиальным, и, тем более, для сравнения двух произвольных узлов.

В [9] В. Хакен, используя теорию нормальных Г. Кнезера [10], предложил алгоритм для распознавания тривиального узла, вьгисления рода произвольного узла, а также определения, является ли данное зацепление разводимым. Х. Шуберт [11], продолжая работу Хакена, построил алгоритм для разложения неразводимого зацепления в связную сумму простых слагаемых. Сушествование и единственность такого разложения для узлов была доказана Шубертом ранее в [12], а на произвольные зацепления этот результат обобщил Й. Хашицуме [13].

Хакен сформулировал также идеи решения общей задачи распознавания зацеплений с помошью распознавания их дополнительных пространств, которые являются так называемыми достаточно большими многообразиями [14], [15]. Значительньй прогресс в реализации программы Хакена был достигнут благодаря развитию теории достаточно больших многообразий в работах $\Phi$. Вальдхаузена [16], Йоганнсона [17], У. Джейко и П. Шэлена [18], а также решению Дж. Хемионом проблемы сопряженности в групшах классов отображений [19]. Проблема алгоритмической классификации неприводимых достаточно больших многообразий (многообразий Хакена) неоднократно объявлялась решенной [20]-[22]. Однако, как указал С.В. Матвеев [23], этих результатов было все еше недостаточно для завершения программы Хакена. Матвеев заделал пробел с помошью более поздних результатов [24], [25]. Подробное описание алгоритма распознавания многообразий Хакена, дающего в частном случае алгоритм распознавания зацеплений, появилось лишш недавно в [26].

Работа выполнена при поддержке Российского фонда фундаментальных исследований (грант № 02-01-00659). 
Таким образом, вопрос о формальной разрешимости задачи сравнения двух данных зацеплений считается решенным. Однако алгоритм в [26] настолько сложен, что превратить его в работаюшую компьютерную программу практически невозможно даже в тех случаях, когда эквивалентность или неэквивалентность данных узлов "очевидна”. Это обстоятельство наводит на мысль, что существующий алгоритм далеко не оптимален, и мотивирует поиск новых подходов к алгоритмической классификации.

Другой подход к алгоритмической классификации многообразий Хакена основан на теореме Тёрстона о гиперболизации [27], [28]. Однако подробное его описание в литературе отсутствует.

В конце 1960-х в работах Г.С. Маканина [29] и Ф. Гарсайда [30] независимо была решена проблема сопряженности в группах кос. Полученные алгоритмы требовали очень большого перебора. Гарсайд указал также и новьй алгоритм для решения проблемы равенства в групе кос, этот алгоритм также был очень медленным. Отметим, что примерно до середины 1980-х годов сложность алгоритмов, не связанных напрямую с решением технических задач, не обсуждалась. Интерес к подобньм вопросам появился в связи с широким распространением персональных компютеров, позволивших применять построенные алгоритмы для дальнейшего исследования. В 1980-х гг. У. Тёрстон сушественно улучшил алгоритм Гарсайда для распознавания кос и получил алгоритм с полиномиальной по длине входа оценкой на время работы [31]. Тёрстон доказал, что групшы кос обладают биавтоматной структурой.

Впоследствии и алгоритм для распознавания классов сопряженности кос был сушественно ускорен [32]-[34].

В конце 1980-х - начале 1990-х Дж. Бирман и У. Менэско стали активно развивать подход Александера-Маркова, основанный на представлении зацеплений замкнутьми косами [35]-[41]. Развитые ими методы привели к построению нового алгоритма для распознавания тривиального узла [42], который, по-видимому, уступает алгоритму Хакена в скорости, но позволяет получать данные, полезные для дальнейшего исследования. Эта теория по-прежнему находится в стадии активной разработки.

В недавней работе [43] автор предложил еще один алгоритм распознавания тривиального узла, принципиальное отличие которого от ранее известных состоит в том, что он не использует дополнительные конструкции такие, как триангулящии дополнительного пространства и расслоенные диски, а работает непосредственно с диаграммами узлов, монотонно их упрошая. Тот же метод позволяет находить разложение зацепления на простые неразводимые слагаемые.

Настояшая работа посвящена обзору результатов, связанных с алгоритмическим распознаванием узлов и зацеплений. По возможности мы приводим ключевые конструкции самих алгоритмов, либо излагаем их основные идеи. При этом мы касаемся далеко не всех алгоритмических аспектов теории узлов. Мы не рассматриваем вопросов, связанных с перечислением узлов, т.е. построением списков узлов, в которых представлен каждый изотопический класс, но не обязательно ровно один раз. По этому поводу см. [44]-[46]. Мы также не обсуждаем вопросов, связанных с алгоритмами вычисления классических инвариантов узлов (исключение составляет род узла). Этому вопросу посвящена, например, работа [47].

Здесь предлагается также доказательство основного результата [43], несколько от- 
личное от первоначального. Это доказательство возникло в результате обсуждения с И. Эголом во время моего визита в университет штата Иллинойс в Чикаго в январе 2003 года.

Мы предполагаем известными стандартные понятия теории узлов, которые можно найти в любом учебнике по узлам, например, [48], [49]: узел, зацепление, связная сумма, несвязная сумма, поверхность Зейферта, род узла, тривиальньй узел. Рассматриваются только ручные узлы. В зависимости от ситуации, мы работаем в гладкой или кусочно-линейной категории, не оговаривая это специально. Все объемлющие изотопии для простоты называются просто изотопиями, поскольку никаких других мы не рассматриваем.

\section{2. Способы представления узлов и зацеплений}

2.1. Выбор комбинаторной модели. Построение любого алгоритма, работающего с топологическими объектами такими, как узлы, естественно начать с выбора некоторого комбинаторного способа задания этих объектов. Вопрос о том, с помощью какой комбинаторной модели лучше представлять узлы, может показаться неважным на первый взгляд. Действительно, все способы, которые мы здесь опишем, “полиномиально эквивалентны” с точки зрения сложности использующих их алгоритмов. А именно, если узел представлен каким-либо из указанных здесь способов, перевести его на другие "язьки" можно за время, полиномиальное по сложности исходного представления. Таким образом, если для одного способа представления узлов какой-либо алгоритм оказался полиномиальным, то полиномиальный алгоритм, решаюший ту же задачу, найдется и для других способов. На сегодняшний день не существует по-настоящему быстрых алгоритмов, распознающих узлы, и алгоритмическая сложность задач, связанных с узлами, обсуждается на уровне принадлежности классам Р и (чаще) NP.

Несмотря на только что сказанное, мы не исключаем, что удачный выбор "комбинаторной модели" для узлов мог бы подсказать более эффективное решение алгоритмических задач теории узлов. Стоит ли напоминать, насколько важным является выбор позиционной системы счисления для построения алгоритмов вьполнения арифметических операций? Возможно, что трудности в построении алгоритмов распознавания узлов связаны, по крайней мере отчасти, с неудачным выбором “системы счисления".

Обозначим через $\mathscr{L}$ множество изотопических классов всех зацеплений. Формально под комбинаторной моделью зацеплений мы понимаем набор $(X, M, \varphi, c)$, где $X-$ некоторое перечислимое множество (элементы которого назьваются диаграммами), $M$ - некоторое перечислимое подмножество в $X \times X$ (элементы которого назьваются элементарными преобразованиями), $\varphi: X \rightarrow \mathscr{L}$ - некоторое отображение и $c: X \rightarrow \mathbb{N}$ - некоторая выгислимая функция (назьваемая функцией сложности) такие, что выполнены следующие условия:

1) сушествует алгоритм, вычисляюший по заданной диаграмме $D$ некоторое зацепление $L_{D}$ из класса $\varphi(D)$ как набор ломаных в $\mathbb{R}^{3}$ с целочисленными вершинами, а также алгоритм, вычис ляющий по заданному зацеплению $L$, представленному набором ломаных в $\mathbb{R}^{3}$, некоторую диаграмму $D$ такую, что $L \in \varphi(D)$ (в частности, отображение $\varphi$ должно быть сюръективно); 
2) равенство $\varphi(D)=\varphi\left(D^{\prime}\right)$ имеет место тогда и только тогда, когда $D \sim D^{\prime}$, где через $\sim$ мы обозначили отношение эквивалентности в $X$, порожденное отношением $M$;

3) для любого $n \in \mathbb{N}$ сушествует лиш конечное число диаграмм $D \in X$ таких, что $c(D) \leqslant n$.

Мы будем пользоваться следуюшими комбинаторными моделями зацеплений.

2.2. Плоские диаграммы. Это - наиболее часто используемый способ представления зацеплений. В качестве диаграмм используются регулярные проекции зацеплений на плоскость, не имеюшие тройных точек. В двойных точках указывается, какая из пересекаюшихся дуг проходит выше. Такие проекции рассматриваются с точностью до изотопии.

В качестве элементарных преобразований выступают движения Райдемайстера, см. рис. 1. Сложность плоской диаграммы $D$ мы определяем как

$$
c(D)=\text { число пересечений в } D+\text { число связных компонент } D \text {. }
$$

Возможность алгоритмически перечислить плоские диаграммы и построить по каждой диаграмме соответствующее зацепление, и наоборот, очевидна. Вьполнение условия 2) в определении комбинаторной модели устанавливается следующей теоремой Райдемайстера.
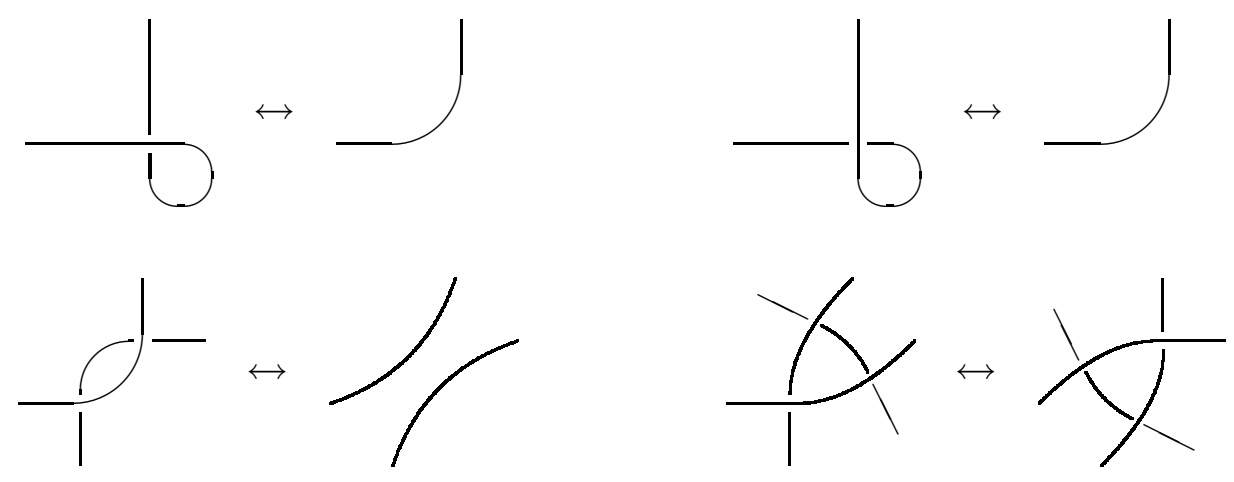

Рис. 1. Движения Райдемайстера

Теорема 2.1 (Райдемайстер [4]). Плоские диаграммы $D$ и $D^{\prime}$ задают изотопные зачепления тогда и только тогда, когда найдется конечная последовательность движений Райдемайстера $D=D_{0} \mapsto D_{1} \mapsto \cdots \mapsto D_{N}=D^{\prime}$.

Все, сказанное вьше, буквально распространяется на случай ориентированных зацеплений. Для этого вместо обычных плоских диаграмм следует рассматривать ориентированные диаграммы зацеплений.

2.3. Замкнутые косы. Косой из $n$ нитей называется одномерное подмногообразие $b \subset\left\{(x, y, z) \in \mathbb{R}^{3} ; 0 \leqslant x \leqslant 1\right\}$, имеюшее $n$ связных компонент (называемых нитями), такое, что

1) пересечение $b$ с плоскостями $x=0$ и $x=1$ состоит из точек $(0, k, 0),(1, k, 0)$, $k=1,2, \ldots, n$ 
2) ограничение координаты $x$ на каждую нить является строго монотонной функцией.

Косы назьваются эквивалентнылми, если они изотопны в $\left\{(x, y, z) \in \mathbb{R}^{3} ; 0 \leqslant x \leqslant 1\right\}$, где предполагается, что плоскости $x=0,1$ при изотопии фиксированы.

Множество изотопических классов кос на $n$ нитях обозначается через $B_{n}$. Для двух кос $b_{1}, b_{2}$ композичия $b_{1} \circ b_{2}$ определяется следуюшим образом:

$$
b_{1} \circ b_{2}=f_{1}\left(b_{1}\right) \cup f_{2}\left(b_{2}\right),
$$

где $f_{1}(x, y, z)=(x / 2, y, z), f_{2}(x, y, z)=((1+x) / 2, y, z)$. Нетрудно видеть, что эта операция корректно задает некоторое умножение в $B_{n}$. В дальнейшем мы всегда рассматриваем косы с точностью до изотопии.

Каждой косе $b \in B_{n}$ сопоставляется ориентированное зацепление $L_{b}$, называемое замыканием косы $b$, как показано на рис. 2.

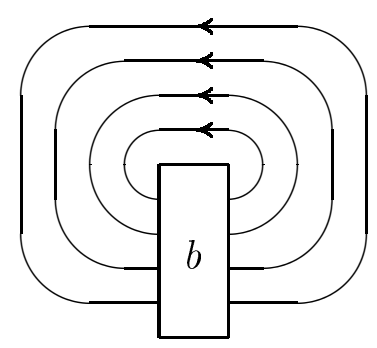

Рис. 2. Замыкание косы $b$

Возможность использования множества всех кос $B_{1} \sqcup B_{2} \sqcup \cdots$ для построения комбинаторной модели ориентированных зацеплений в смысле данного вьше определения вытекает из следующих трех результатов.

ТЕОРема 2.2 (Александер [5]). Любое ориентированное зачепление изотопно зацеплению вида $L_{b}$, где $b$ - некоторая коса.

Эта теорема была сформулирована Александером в несколько иных терминах еще до того, как группы кос были введены Артином.

Теорема 2.3 (Артин [8]). Для каждого $n \leqslant 1$ множество $B_{n}$ кос вместе $c$ операчией композичии образует группу. Эта группа может быть задана набором образующих $\sigma_{i}, i=1, \ldots, n-1$, показанных на рис. 3, и определяющими соотношениями:

$$
\begin{gathered}
\sigma_{i} \sigma_{j}=\sigma_{j} \sigma_{i}, \quad 0<i<j-1<n-1, \\
\sigma_{i} \sigma_{i+1} \sigma_{i}=\sigma_{i+1} \sigma_{i} \sigma_{i+1}, \quad 0<i<n-1 .
\end{gathered}
$$

В әруппе $B_{n}$ разрешима проблема равенства. 


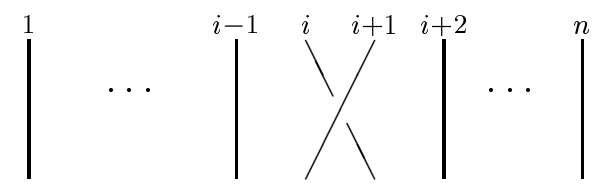

Рис. 3. Коса $\sigma_{i}$ (ось $O x$ направлена вниз)

Набор элементарных преобразований, порождаюших отношение изотопности для замкнутых кос, дан в следующей теореме (ее подробное доказательство впервые появилось в [50].

Теорема 2.4 (А.А. Марков [6]). Замыкания кос $b_{1}$ и $b_{2}$ изотопны как ориентированные зацепления в $\mathbb{R}^{3}$ тогда и только тогда, когда их можкно получить друг из друга конечной последовательностью следующих операций:

1) сопряжение: $b \mapsto c b c^{-1}, b, c \in B_{n}$;

2) стабилизачия: $b \mapsto \iota_{n}(b) \sigma_{n}^{ \pm 1}, b \in B_{n}$;

3) дестабилизачия: операция, обратная к стабилизачии.

Здесь $\iota_{n}$ обозначает вложсение $\iota_{n}: B_{n} \rightarrow B_{n+1}$, заданное отождествлением соответствуюших образующих: $\iota_{n}\left(\sigma_{i}\right)=\sigma_{i}$.

Операции, перечисленные в этой теореме, называются движениями Маркова, при этом сопряжение часто называется первым, а стабилизация или дестабилизация вторым движением Маркова.

Замыкания кос не используются как комбинаторная модель для неориентированных зацеплений, поскольку для операции обращения ориентации одной из компонент зацепления не известно хорошего описания в терминах кос.

Напрактике косы обычно представляются с помощью слов в образуюших $\sigma_{i}$, поэтому на самом деле в данной комбинаторной модели зацепления представляются парами вида $(n, w)$, где $n$ - натуральное число, а $w$ - слово в образуюших $\sigma_{i}, i=1, \ldots, n-1$. В качестве сложности такого представления мы выбираем следуюшую функцию:

$$
c(n, w)=n+|w|,
$$

где $|w|$ - это длина слова $w$. Для косы $b \in B_{n}$ ее сложность $c(b)$ определяется как

$$
c(b)=\min _{w}(n+|w|),
$$

где минимум берется по всем словам $w$, представляюшим $b$.

2.4. Прямоугольные диаграммы. Этот способ представления зацеплений был введен автором недавно в [43]. Прямоугольной диаграммой сложности $n$ мы будем называть набор $R$ целочисленных точек вида $(i, j), 1 \leqslant i, j \leqslant n$, на плоскости $\mathbb{R}^{2}$ такой, что на каждой прямой $x=k$ и $y=k, k=1, \ldots, n$, имеются ровно две точки из $R$. Ориентированной прямоугольной диаграммой сложности $n$ назьвается прямоугольная диаграмма $R$ сложности $n$ с фиксированным разбиением $R=R_{-} \cup R_{+}$ таким, что каждая прямая $x=k$ и $y=k, k=1, \ldots, n$, содержит ровно по одной точке из $R_{-}$и $R_{+}$. 
Прямоугольной диаграмме $R$ мы сопоставляем зацепление

$$
L_{R}=\bigcup_{(i, j) \in R}[(i, 0,1),(0, j, 0)],
$$

где через $[A, B]$ обозначен отрезок, соединяюший точки $A$ и $B$. Если $R$ - ориентированная прямоугольная диаграмма, то отрезки (2), соответствующие точкам из $R_{+}$, ориентируются по возрастанию третьей координаты, а отрезки, соответствуюшие точкам из $R_{-},-$наоборот.

Нетрудно получить следуюшие оценки числа прямоугольных диаграмм данной сложности.

ПРЕДЛОЖЕНИЕ 2.1. Число ориентированных прямоугольных диаграмм зачеплений сложности п равно

$$
(n !)^{2} \cdot \sum_{k=0}^{n} \frac{(-1)^{k}}{k !} .
$$

Число (неориентированных) прямоугольных диаграмм узлов сложсности п равно

$$
\frac{(n-1) ! n !}{2}
$$

Отсюда следует, что число $N(n)$ (неориентированных) прямоугольных диаграмм зацеплений сложности $n$ удовлетворяет неравенствам

$$
(n-1) ! n ! \leqslant 2 N(n) \leqslant(n !)^{2} \cdot \sum_{i=0}^{n} \frac{(-1)^{k}}{k !} .
$$

Набор необходимых элементарных преобразований указан в следующей теореме.

ТеОрема 2.5. Любое зацепление можно представить прямоугольной диаграммой.

Две прямоугольные диаграммы задают изотопные зацепления тогда и только тогда, когда одну мохно получить из другой конечной последовательностью преобразований следующего вида:

1) циклическая перестановка: $(i, j) \mapsto(i+1, j), i=1, \ldots, n-1,(n, j) \mapsto(1, j)$, или $(i, j) \mapsto(i, j+1), j=1, \ldots, n-1,(i, n) \mapsto(i, 1) ;$

2) вертикальная рокировка: четыре точки $(i, j),(i, k),\left(i+1, j^{\prime}\right),\left(i+1, k^{\prime}\right)$, для которых пары $(j, k) u\left(j^{\prime}, k^{\prime}\right)$ незачепленьи, заменяются на $\left(i, j^{\prime}\right),\left(i, k^{\prime}\right)$, $(i+1, j),(i+1, k)$

3) горизонтальная рокировка: четыре точки $(i, k),(j, k),\left(i^{\prime}, k+1\right),\left(j^{\prime}, k+1\right)$, для которых пары $(i, j)$ и $\left(i^{\prime}, j^{\prime}\right)$ незачеплены, заменяются на $\left(i^{\prime}, k\right),\left(j^{\prime}, k\right)$, $(i, k+1),(j, k+1)$;

4) стабилизачия: диаграмма $R$ сложсноти $n$ такая, что $(n, n) \in R$, заменяется на диаграмму сложности $n+1$, которая получается из $R$ одной из следующих четырех замен:

$(n, n) \mapsto(n, n+1),(n+1, n+1),(n+1, n)$,

$(i, n) \mapsto(i, n+1),(n+1, n+1),(n+1, n)$,

$(n, j) \mapsto(n+1, j),(n+1, n+1),(n, n+1)$,

$(i, n),(n, j) \mapsto(i, n+1),(n, n+1),(n+1, n),(n+1, j)$

5) дестабилизачия: операчия, обратная к стабилизачии. 
Здесь под незачепленны.ми парами $(i, j),(k, l)$ мы понимаем такие, в которых числа $i, j, k, l$ попарно различны и коэффищиент зацепления которых в $\mathbb{R}$ равен нулю в обычном гомологическом смысле. Это равносильно условию

$$
-\infty<\frac{(i-j)(k-l)}{(i-l)(k-j)}<1, \quad i \neq j, \quad k \neq l .
$$

Теорема 2.5 в несколько другой формулировке содержится в [43]. Она элементарно сводится к теореме Райдемайстера. Ранее утверждение, фактически равносильное теореме 2.5, но сформулированное в геометрических терминах, появилось в [51].

Объясним происхождение прямоугольных диаграмм. Назовем геометрической прямоугольной диаграммой зацепления обычную плоскую диаграмму зацепления $D$, удовлетворяющую следуюшим ограничениям:

1) $D$ состоит из вертикальных и горизонтальных отрезков, никакие два из которых не лежат на одной прямой (мы называем их ребрами, а их концы-вершинами диаграммы $D$ );

2) в каждом пересечении вертикального и горизонтального ребра вертикальное проходит сверху, см. рис. 4.

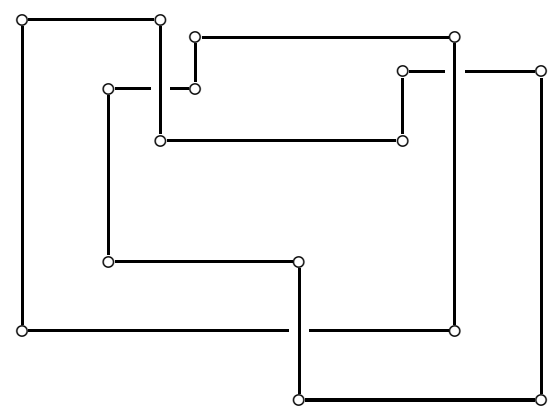

Рис. 4. Геометрическая прямоугольная диаграмма

Определенные выше прямоугольные диаграммы - это комбинаторные классы геометрических прямоугольных диаграмм, т.е. геометрические прямоугольные диаграммы, рассматриваемые с точностью до гомеоморфизмов плоскости вида $(x, y) \mapsto(f(x), g(y))$, где $f, g$ - возрастающие функщии. Чтобы увидеть это, занумеруем вертикальные ребра некоторой геометрической прямоугольной диаграммы $D$ слева направо, а горизонтальные - снизу вверх и рассмотрим множество $R$ пар $(i, j)$ таких, что вертикальное ребро с номером $i$ и горизонтальное с номером $j$ имеют обший конец. Получим прямоугольную диаграмму, задающую комбинаторный тип диаграммы $D$. Нетрудно проверить, что зацепление $L_{R}$ будет изотопно тому, которое задается плоской диаграммой $D$.

Прямоугольные диаграммы были введены автором для удобного изображения книжных зацеплений, см. раздел 6, однако можно интерпретировать эту ситуацию и так, что книжные зацепления являются удобным инструментом для изучения свойств прямоугольных диаграмм. 
2.5. Переход от одного задания к другому. Указанные способы представления зацеплений легко преобразуются друг в друга. Очевидно, как построить алгоритмы, преобразуюшие косу или прямоугольную диаграмму в плоскую диаграмму. Доказательство теоремы Александера фактически состоит в построении алгоритма преобразования ориентированной плоской диаграммы в косу. Наиболее простой такой алгоритм был предложен П. Вожелем в [52]. Здесь мы приведем алгоритмы для преобразования плоской диаграммы в прямоугольную и прямоугольной диаграммы в косу. Заметим, что этот подход дает новьй, методически очень простой, способ доказательства теорем Александера и Маркова.

ПРЕДЛОЖЕНИЕ 2.2. Для любой плоской диаграммы сложсности $n$ найдется прямоугольная диаграмма сложности $\leqslant 2 n$, задающая изотопное зачепление.

Идея доказательства проста и проиллюстрирована на рис. 5. Сначала берется некоторая горизонтальная прямая $\ell$, проходяшая ниже данной диаграммы. Затем пересечения диаграммы одно за другим проталкиваются вниз под $\ell$. При этом каждый раз добавляется по два вертикальных ребра, и еше два добавляются на каждую связную компоненту исходной диаграммы. Когда выше $\ell$ не остается пересечений, все находяшиеся там дуги можно продеформировать в объединение вертикальных и горизонтальных отрезков без добавления дополнительных вертикальных ребер.
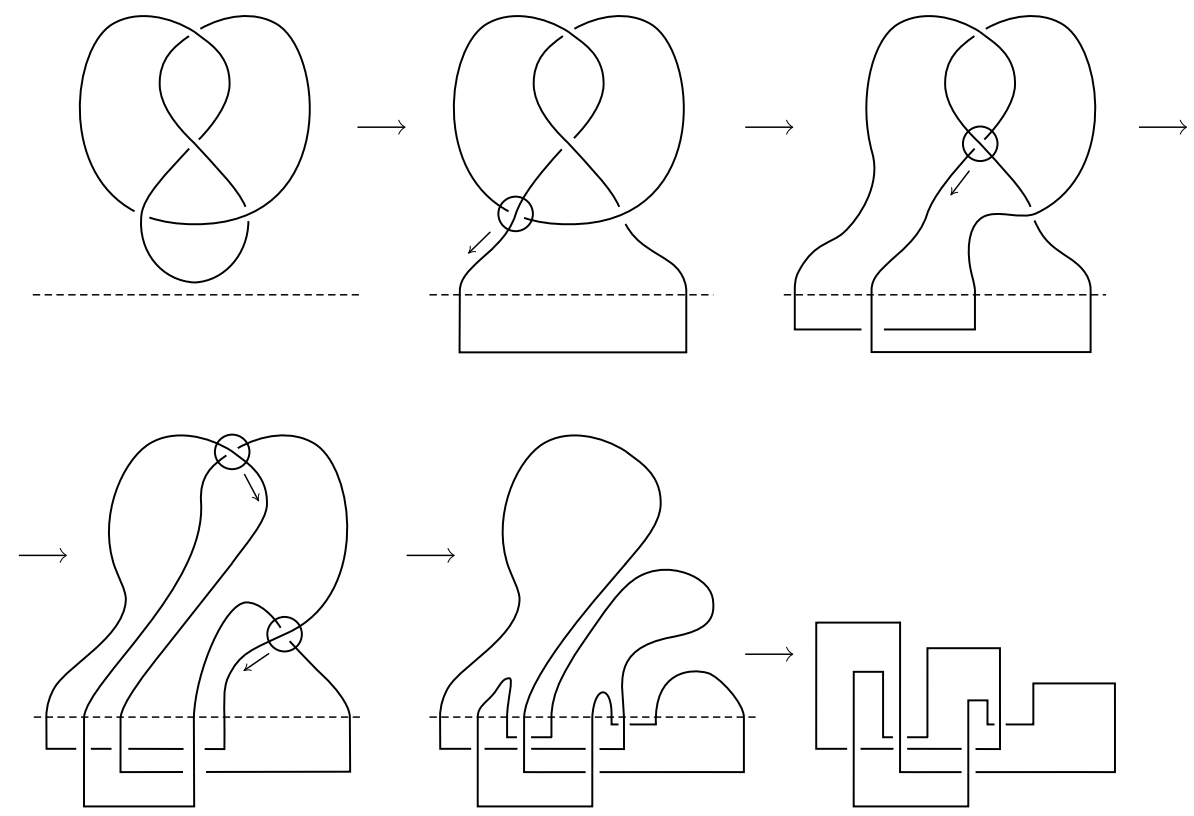

Рис. 5. Превращение плоской диаграммы в прямоуголшную

На рис. 6 показано, как по ориентированной прямоугольной диаграмме $R$ построить косу $b$ такую, что $L_{R} \sim L_{b}$. Для этого каждое горизонтальное ребро, ориентация которого противоположна ориентации горизонтальной оси, заменяется на два “дополнительных” отрезка. При этом во всех новых пересечениях вертикальные отрезки сно- 


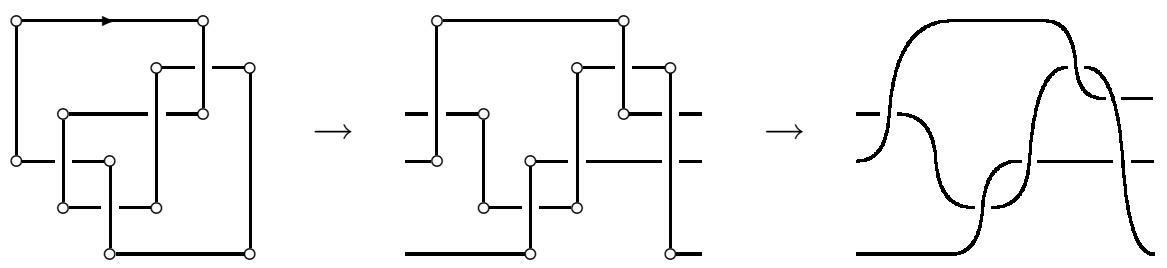

Рис. 6. Превращение ориентированной прямоугольной диаграммы в косу

ва проходят над горизонтальными. После малого шевеления получается регулярная проекция косы $b$.

Приведем краткую справку о том, как меняется сложность представления (в худшем случае) при переходе от одной комбинаторной модели к другой:

\begin{tabular}{|c|c|}
\hline преобразование & изменение сложности \\
\hline коса $\mapsto$ плоская диаграмма & $n \mapsto n$ \\
\hline плоская диаграмма $\longmapsto$ прямоугольная диаграмма & $n \mapsto 2 n$ \\
\hline прямоугольная диаграмма $\longmapsto$ плоская диаграмма & $n \mapsto n^{2} / 4$ \\
\hline прямоугольная диаграмма $\mapsto$ коса & $n \mapsto n^{2} / 4$ \\
\hline
\end{tabular}

Не указанные здесь преобразования можно получить композицией указанных.

\section{3. Метод Хакена}

Пусть $M$ - трехмерное компактное многообразие (возможно, с краем), наделенное триангулящией $T$. Собственно вложенная поверхность $F \subset M$ называется нормальной по отношению к триангуляции $T$, если пересечение $F \cap \Delta$ поверхности $F$ с каждьм 3 -симплексом $\Delta \in T$ состоит из треугольников и четырехугольников, вершины которых лежат на попарно различных ребрах симплекса $\Delta$, см. рис. 7 . Такие треугольники и четырехугольники назьваются элементарными дисками. Два элементарных диска принадлежат одному muny, если их границы пересекают одни и те же ребра. Всего имеется $7 t$ типов элементарных дисков, где $t$ - общее число 3 -симплексов в триангулящии $T$.

ЗАмЕчАниЕ. Сушествует другой, “дуальньй”, подходк определению нормальных поверхностей. Для него вместо триангулящи многообразия используется разбиение на ручки. С формальной точки зрения эти подходы совершенно эквивалентны. С точки зрения практического применения подход, основанньй на разбиении на ручки, несколько эффективнее: системы уравнений для нахождения фундаментальных поверхностей (см. ниже) получаются проще. Однако речь здесь идет не о качественном улучшении асимптотической сложности алгоритмов, а об улучшении некоторых констант в оценке сложности. В конкретных примерах малой сложности обычно лучше воспользоваться разбиением на ручки. Однако описание метода становится проще, если пользоваться триангулящиями, что мы и будем делать. 


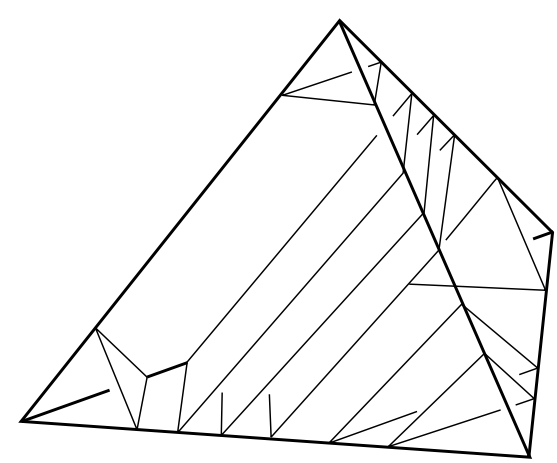

Рис. 7. Пересечение $F \cap \Delta$

Нормальные поверхности были введены Х. Кнезером [10], которьй использовал их для оценки сверху числа попарно непараллельных и непересекающихся несжимаемых сфер в трехмерном многообразии.

Для задания нормальной поверхности Хакен предложил занумеровать типы элементарных дисков и сопоставить каждой нормальной поверхности $F$ целочисленный вектор $x(F)=\left(x_{1}, \ldots, x_{7 t}\right), i$-я координата которого равна количеству элементарных дисков $i$-го типа, составляюших поверхность $F$. Величины $x_{i}(F)$ называются нормальными координатами поверхности $F$. Вектор $x(F)$ однозначно определяет поверхность $F$ с точностью до изотопии в классе нормальных поверхностей, но не все наборы целых чисел $x \in \mathbb{Z}^{7 t}$ соответствуют нормальным поверхностям. Можно выписать систему уравнений и неравенств, задающую подмножество всех векторов вида $x(F)$. Это делается следуюшим образом.

Во-первых, чтобы вектор $x$ задавал некоторую нормальную поверхность, должно, очевидно, выполняться условие

$$
x_{i} \geqslant 0, \quad i=1, \ldots, 7 t \text {. }
$$

Во-вторых, пусть два 3-симплекса $\Delta_{1}, \Delta_{2} \in T$ имеют общую 2-грань $f$. Пересечение $F \cap f$ состоит из дуг, концы каждой из которых лежат на разных сторонах грани $f$. Всего имеется три типа дуг. Количество дуг каждого типа можно вычислить, зная число элементарных дисков в $\Delta_{1}$, оно будет иметь вид $x_{i}+x_{j}$ (ровно два типа дисков в одном 3-симплексе вносят вклад в количество дуг данного типа). Точно так же, его можно выразить через число элементарных дисков в $\Delta_{2}$, скажем, $x_{k}+x_{l}$. Таким образом, для каждой 2-грани, не лежащей на крае $\partial M$, следует вьписать три уравнения вида

$$
x_{i}+x_{j}=x_{k}+x_{l} .
$$

Наконец, некоторые типы дисков несовместимы в том смыле, что любые диски этих типов обязательно пересекаются, поэтому они не могут одновременно входить в нормальную поверхность. А именно, в каждом 3-симплексе четырехугольные элементарные диски попарно несовместимы. Поэтому векторы $x(F)$ удовлетворяют также условИю

$$
x_{i} x_{j}=0,
$$


где $i, j$ - номера любой пары различных типов четырехугольных элементарных дисков, лежащих в одном 3-симплексе.

Нетрудно доказать следующее утверждение.

ПРЕДЛОЖЕНИЕ 3.1. Вектор $x \in \mathbb{Z}^{7 t}$ задает нормальную поверхность тогда и только тогда, когда он удовлетворяет условиям (3)-(5).

Фундаментальным решением системы (3), (4) называется такое решение $x \in \mathbb{Z}^{7 t}$, что $x$ нельзя нетривиальным образом разложить в сумму $x=x_{1}+x_{2}$ двух других решений. Нормальные поверхности $F$, для которых $x(F)$ является фундаментальным решением системы (3), (4) также назьваются фундаментальными. Следуюшее, легко доказываемое, утверждение играет ключевую роль для метода Хакена.

Лемма 3.1. Множество фундаментальных решений системы (3), (4) конечно и может бить найдено алгоритмически.

Любое нефундаментальное решение является линейной комбинацией фундаментальных с неотрицательными цельми коэффициентами.

Хакен доказал следующие два утверждения.

ПРЕДЛОЖЕНИЕ 3.2. Пусть $M$ - триангулированное дополнение $к$ открытой трубчатой окрестности произвольного узла $K \subset S^{3}$. Тогда среди набора фундаментальных поверхностей для $M$ найдется поверхность Зейферта узла $K$ минимально возможкного рода.

ПРЕДЛОЖЕНИЕ 3.3. Пусть $M$ - триангулированное дополнение $\kappa$ открытой трубчатой окрестности произвольного зачепления $L \subset S^{3}$. Eсли зацепление $L$ разводимо, то среди набора фундаментальных поверхностей для $M$ найдется сфера, разлагающ, $L$ в нетривиальную несвязную сумму.

Тем самым в [9] была доказана следуюшая теорема.

ТЕОРема 3.1. Существует алгоритм, который по заданному узлу за конечное время вичисляет его род.

Существует алгоритм, который по заданному зацеплению за конечное время отвечает, является ли әто зачепление разводимым и, если да, указывает его разложсение в несвязную сумму неразводимых зацеплений.

Для метода Хакена не имеет принципиального значения, каким именно образом задан узел или зацепление. Первьй шаг алгоритма Хакена состоит в нахождении триангулящии дополнения к трубчатой окрестности данного зацепления. Затем выписывается система уравнений и неравенств (3), (4) и находятся ее фундаментальные решения. Среди них отбираются те, которые удовлетворяют условию (5). Наконец, среди найденных фундаментальных поверхностей отбирается: поверхность Зейферта наименьшего рода в случае нахождения рода узла или разбиваюшая сфера в случае проверки разводимости данного зацепления.

Результат Хакена позволяет, в частности, построить алгоритм распознавания тривиального узла, поскольку это единственньй узел рода нуль.

Следуя методу Хакена, Шуберт доказал в [11] следуюший результат. 
ТеОрема 3.2. Существует алгоритм, который по заданному неразводимому зацеплению отвечает, является ли оно простым и, если нет, указывает его разложсение в связную сумму простых зачеплений.

Доказательство снова основано на том, что поверхность, которая нам нужна, в данном случае факторизуюшую сферу - всегда можно найти среди фундаментальных.

Таким образом, задача алгоритмической классификации узлов и зацеплений сводится к задаче сравнения двух простых неразводимых зацеплений. В [14], [15] Хакен предложил программу решения последней, которая была завершена лишш недавно в книге Матвеева [26]. Полученньй в итоге алгоритм чрезмерно сложен, так что не имеет смысла обсуждать вопрос о его практической реализации. Кроме метода нормальных поверхностей, для его построения понадобились результаты работ [17]-[19], [24], [25].

Укажем очень кратко и поверхностно идеи и основные трудности при построении только что упомянутого алгоритма сравнения зацеплений. Мы будем следовать интерпретации Матвеева. Прежде всего, для данных зацеплений строятся триангуляции (или разбиения на ручки) их дополнительных пространств, пусть $M_{1}, M_{2}$. Как известно, дополнительное пространство не содержит полной информации о зацеплении, но если на каждой компоненте его границы указать меридиан и параллель, то полученное многообразие с узором на крае однозначно определяет соответствующее зацепление.

Если данные зацепления неразводимы, просты и не являются тривиальньпи узлами, то многообразия $M_{1}, M_{2}$ принадлежат классу так назьваемых многообразий Хакена (неприводимых, гранично неприводимых и содержаших некоторую несжимаемую поверхность).

ТЕОРема 3.3 [26]. Существует алгоритм, который по двум ориентированным многообразиям Хакена $M_{1}, M_{2}$ с узором на крае отвечает, существует ли сохраняющий ориентацию гомеоморфизм $M_{1} \rightarrow M_{2}$, переводящий узор на $M_{1}$ в узор на $ә M_{2}$.

Это - более общее утверждение, чем требуется для алгоритмического сравнения зацеплений.

Для сравнения двух многообразий Хакена в каждом из них специальным образом находится двумерный полиэдр, разбиваюший многообразие на трехмерные шары, после чего найденные разбиения сравниваются. Утверждается, что процедуру нахождения разбиения можно построить таким образом, чтобы результат не зависел от триангуляции многообразия.

Разбивающие полиэдры находятся с помощью построения так называемых иерархий. Иерархия в многообразии Хакена $\mathrm{M}$ - это последовательность $\partial M=P_{0} \subset$ $P_{1} \subset P_{2} \subset \cdots \subset P_{m}$ двумерных полиэдров, вложенных в $M$, в которой каждый следуюший, $P_{i+1}$, получается из предыдушего, $P_{i}$, добавлением некоторой поверхности $F_{i} \subset M \backslash P_{i}$, а последний полиэдр $P_{m}$ разбивает $M$ на клетки. На каждом шаге это можно сделать несколькими способами, поэтому сначала алгоритм находит большое семейство разбиений многообразия $M$, а потом выбирает среди них наименьшее 
по отношению к лексикографическому порядку, который возникает при фиксировании способа кодирования разбиений.

Основная трудность при алгоритмическом построении канонического разбиения многообразия Хакена состоит в формулировке правила, по которому на каждом шаге к полиэдру $P_{i}$ будет добавляться поверхность $F_{i}$. Это правило должно удовлетворять следуюшим условиям:

1) любая иерархия обрывается за конечное число шагов, не превьшающее некоторое $N$, зависящее от многообразия $M$ и выбранной триангуляции;

2) для любого уже построенного полиэдра $P_{i}$ наше правило должно давать лишш конечное число возможностей для добавления поверхности $F_{i}$;

3) набор построенных разбиений, рассматриваемых с точностью до гомеоморфизма многообразия $M$, должен совпадать с некоторым классом разбиений, определяемых топологически, без ссылки на триангулящию многообразия $M$.

При построении каждой следующей поверхности $F_{i}$ используется метод нормальных поверхностей, но гораздо более сложньм образом, чем в случае алгоритмов Хакена и Шуберта. Если, скажем, брать в качестве $F_{i}$ всевозможные фундаментальные поверхности в $M \backslash P_{i}$, то невьполненным окажется свойство 3), так как набор фундаментальных поверхностей зависит от триангуляции. Если же перебирать в качестве $F_{i}$ всевозможные несжимаемые поверхности (которые всегда можно произотопировать в нормальные), то невьполненным будет условие 2), поскольку несжимаемых поверхностей может быть бесконечное число, и т. д.

Одной из существенных трудностей в реализации программы Хакена явилась необходимость решать проблему сопряженности в группах классов отображений компактных поверхностей. Эта проблема была решена Хемионом в [19], после чего задача алгоритмического распознавания зацеплений считалась решенной. Однако несколько лет назад Матвеев указал, что результатов и техники Хемиона на самом деле недостаточно [23]. А именно, в некоторых случаях для построения иерархии нужно решить следуюшую задачу: для элементов $a, b, a^{\prime}, b^{\prime}$ групшы классов отображений некоторой поверхности найти элемент $c$ такой, что $a^{\prime}=c a c^{-1}$ и $b^{\prime}=c b c^{-1}$, или доказать, что он не сушествует. Решение Хемиона проблемы сопряженности в группах классов отображений не обобщается на этот случай. Матвеев преодолел эту трудность, используя теорию Тёрстона [24], [25] диффеоморфизмов поверхностей, которая была опубликована уже после упомянутой работы Хемиона.

\section{4. Геометризация}

Сушествует иной подход к распознаванию зацеплений, также основанньй на рассмотрении дополнительного пространства. Его идея, принадлежащая Тёрстону, состоит в нахождении на данном многообразии Хакена $M$ геометрической структурь вместо разбиения $M$ на клетки. Если в $M$ имеются несжимаемые торы, то сначала на $M$ находится так называемое $J S J$-разложсение - разрезание многообразия $M$ вдоль торов на зейфертовы многообразия и так называемые простые (simple) многообразия [17], [18], [53]. Нахождение JSJ-разложения и установление структуры зейфертовых кусков происходит так же, как и в методе Хакена. На простых же кусках находится гиперболическая структура, которая существует по теореме Тёрстона о гиперболизации. Доказательство этой теоремы см. в [28]. 
Так же, как и алгоритм построения иерархий, алгоритм для нахождения гиперболической структуры на простом многообразии Хакена очень сложен. В частности, он требует решения систем алгебраических уравнений, задающих пространство представлений группы $\pi_{1} M$ в $P S L(2, \mathbb{C})$. Однако замена "точных" алгебраических вычислений на приближенные не приводит к ошибкам в примерах малой сложности, давая возможность сушественно упростить процедуру. На этом основаны программы SNAPPEA и KNOTSCAPE, позволившие, в частности, перечислить без повторений все простые узлы с $\leqslant 16$ пересечениями на диаграмме.

Подробного изложения алгоритма, основанного на геометризации, в литературе также не существует, можно найти лишш описание идей его построения, см., напримep, [54].

\section{5. Алгоритмы для кос}

5.1. Проблема равенства. Использование кос как инструмента для изучения узлов и зацеплений мотивировано тем, что группы кос обладают рядом замечательных свойств и хорошо поддаются изучению. На сегодняшний день сушествует много различных алгоритмов распознавания кос, в том числе очень быстрых. Ниже мы опишем некоторые из них, однако наш список заведомо неполон.

Проблема равенства для групп кос была решена еше Артином в тех же работах $[7],[8]$, где групшы кос были определены. Значительная часть рассуждений Артина была геометрической. Алгебраическое изложение его теории было дано в [55] и [56]. Из работы Артина извлекается на самом деле два алгоритма для решения проблемы равенства в групе кос. Один из этих алгоритмов приводит данную косу к некоторому специальному виду, которьй может быть экспоненциально сложен по длине изначального представления косы. Мы не будем здесь его описьвать, а приведем лишш второй алгоритм, основанный на схожих идеях.

Алгоритм Артина использует утверждение, которое на современном языке формулируется так.

TEOPEма 5.1. Группа кос $B_{n}$ изоморфна группе классов отображений $\operatorname{MCG}\left(D^{2}, n\right)$ диска $D^{2}$ с $n$ отмеченными точками.

Группа $M C G\left(D^{2}, n\right)$ естественньм образом действует автоморфизмами на фундаментальной группе диска $D^{2}$ с $n$ проколами, т.е. на свободной группе $F_{n}$ от $n$ образуюших $u_{1}, u_{2}, \ldots, u_{n}$. В стандартных образуюших $\sigma_{i}$ групшы $B_{n}$ это действие записывается следуюшим образом (см. рис. 8):

$$
\begin{aligned}
& \sigma_{i} \cdot u_{j}=u_{j}, \text { если } j \neq i, i+1, \\
& \sigma_{i} \cdot u_{i}=u_{i+1} \\
& \sigma_{i} \cdot u_{i+1}=u_{i+1}^{-1} u_{i} u_{i+1} .
\end{aligned}
$$

Если данные две косы $b_{1}, b_{2}$ заданы словами в $\sigma$-образуюших, то равенство $b_{1}=b_{2}$ равносильно $b_{1} \cdot\left(u_{1}, \ldots, u_{n}\right)=b_{2} \cdot\left(u_{1}, \ldots, u_{n}\right)$. Наборы же $b_{1,2} \cdot\left(u_{1}, \ldots, u_{n}\right)$ можно вьчислить по формулам (6) и затем сравнить. Сравнение возможно, поскольку эти строки состоят из элементов свободной группы, а в ней проблема равенства тривиальна. 


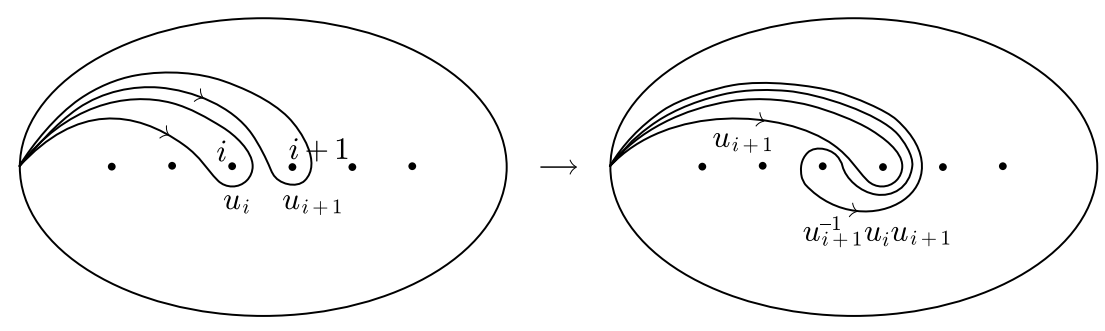

Рис. 8. Действие $\sigma_{i}$ на образующие свободной группь

Несмотря на кажущуюся простоту этого алгоритма, применение его на практике затруднительно, поскольку длины слов $b \cdot u_{i}$ могут расти экспоненциально с ростом длины косы $b$. Мы используем его как поучительньй пример того, как переход к другой “системе счисления" делает алгоритм из экспоненциального полиномиальным.

Для элемента $x \in F_{n}$, записанного в виде слова в образующих $u_{i}$, обозначим через $\bar{x}$ слово, полученное из $x$ следуюшими заменами:

$$
\begin{aligned}
u_{1} & \mapsto v_{1} v_{3} v_{2}^{-1} v_{1}^{-1}, \\
u_{2} & \mapsto v_{1} v_{2} v_{4} v_{6} v_{5}^{-1} v_{4}^{-1} v_{2}^{-1} v_{1}^{-1}, \\
& \ldots \\
u_{i} & \mapsto\left(v_{1} v_{2} v_{4} \ldots v_{3 i-5} v_{3 i-4} v_{3 i-2}\right) v_{3 i} v_{3 i-1}^{-1}\left(v_{1} v_{2} v_{4} \ldots v_{3 i-5} v_{3 i-4} v_{3 i-2}\right)^{-1} .
\end{aligned}
$$

Через $|x|_{j}$ обозначим количество вхождений букв $v_{j}^{ \pm 1}$ в слово $\bar{x}$ после всевозможных сокращений $v_{i}^{ \pm 1} v_{i}^{\mp 1} \mapsto \varnothing$. Через $s(x)$ обозначим следующую последовательность целых чисел длины $2 n$ :

$$
s(x)=\left(\frac{|x|_{2}-|x|_{3}}{2}, \frac{|x|_{1}-|x|_{4}}{2}, \ldots, \frac{|x|_{3 n-1}-|x|_{3 n}}{2}, \frac{|x|_{3 n-2}-|x|_{3 n+1}}{2}\right) .
$$

ЛЕмма 5.1. Если әлементы $x_{1}, \ldots, x_{k} \in F_{n} \cong \pi_{1}\left(D^{2} \backslash\left\{P_{1}, \ldots, P_{n}\right\}\right)$ можнно представить попарно непересекающимися петлями в $D^{2} \backslash\left\{P_{1}, \ldots, P_{n}\right\}$, то они однозначно с точностью до ориентачии восстанавливаются по $s\left(x_{1}\right)+\cdots+$ $s\left(x_{k}\right) \in \mathbb{Z}^{2 n}$.

Идея доказательства состоит в следуюшем. Разрежем диск, как показано на рис. 9. Для петли $x \in F_{n}$ с базовой точкой, отмеченной $*$, числа $|x|_{i}$ указывают количество пересечений петли $x$ c $i$-м разрезом при условии, что все "лишние" пересечения удалены. Нетрудно показать, что по количеству пересечений с указанными разрезами набор несамопересекающихся попарно непересекаюшихся петель можно восстановить, но этот набор не может быть произвольным. Несколько меньший набор, указанньй в лемме 5.1, получается "выбрасьванием лишней информации".

Набор образующих $u_{1}, \ldots, u_{n}$ можно представить попарно непересекаюшимися петлями. Из соображений симметрии мы выбираем несколько другой набор образующих: $\left(u_{1}, u_{1} u_{2}, \ldots, u_{1} \ldots u_{n}\right)$, также обладающий этим свойством. Имеем

$$
s\left(u_{1}, u_{1} u_{2}, \ldots, u_{1} \ldots u_{n}\right)=(0,1, \ldots, 0,1) .
$$




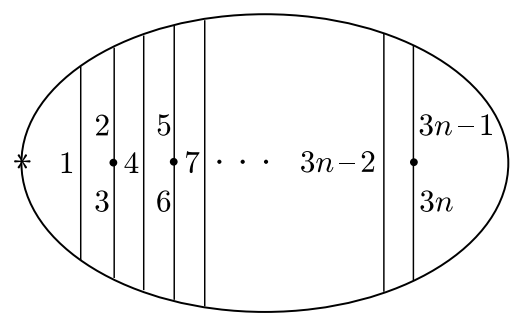

Рис. 9. Разрезание диска $D^{2}$ на “треугольники”

Возможность использования такого кодирования набора образуюших групшы $F_{n}$ появляется благодаря следуюшей лемме (доказательство см. в [57; гл. 8]).

ЛЕмма 5.2. Если $s\left(x_{1}, \ldots, x_{k}\right)=\left(a_{1}, b_{1}, \ldots, a_{n}, b_{n}\right), \operatorname{mos} s\left(\sigma_{i} \cdot x_{1}, \ldots, \sigma_{i} \cdot x_{k}\right)=$ $\left(a_{1}^{\prime}, b_{1}^{\prime}, \ldots, a_{n}^{\prime}, b_{n}^{\prime}\right)$, где $a_{j}^{\prime}=a_{j}, b_{j}^{\prime}=b_{j}$ при $j \neq i, i+1, u$

$$
\begin{aligned}
a_{i}^{\prime} & =a_{i}+\left(\delta^{+}+b_{i}\right)^{+}, \\
a_{i+1}^{\prime} & =a_{i+1}-\left(\delta^{+}-b_{i+1}\right)^{+}, \\
b_{i}^{\prime} & =b_{i}-\left(-\delta^{\prime}\right)^{+}+\delta^{+}, \\
b_{i+1}^{\prime} & =b_{i+1}+\left(-\delta^{\prime}\right)^{+}-\delta^{+},
\end{aligned}
$$

где мы использовали обозначения: $\delta=a_{i+1}-a_{i}, \delta^{\prime}=a_{i+1}^{\prime}-a_{i}^{\prime}, a^{+}=\max (0, a)$.

Таким образом, мы имеем легко вычислимое теоретико-множественное вложение $B_{n} \rightarrow \mathbb{Z}^{2 n}$, заданное формулой

$$
b \mapsto b \cdot(0,1, \ldots, 0,1),
$$

где действие образуюших $\sigma_{i}$ на $\mathbb{Z}^{2 n}$ дается формулами (7). Это дает простой и быстрьй алгоритм распознавания кос. Этот алгоритм был предложен автором в [58], см. также [59], [57]. Аналогичньй алгоритм независимо был построен в [60].

Важньй набор инструментов для изучения групп кос был введен Гарсайдом в [30], где с их помощью было дано решение проблемы равенства, принципиально отличаюшееся от решения Артина, а также решение проблемы сопряженности в группе кос.

Гарсайд рассмотрел моноид $B_{n}^{+}$, заданный образующими $\sigma_{i}, i=1, \ldots, n-1$, и теми же соотношениями (1), что и группа кос. Его элементы называются положительными косами. Гарсайд рассмотрел также следуюший элемент, назьваемьй фундаментальной косой:

$$
\Delta=\left(\sigma_{1} \sigma_{2} \cdots \sigma_{n-1}\right)\left(\sigma_{1} \sigma_{2} \cdots \sigma_{n-2}\right) \cdots \sigma_{1} .
$$

Квадрат $\Delta^{2}$ этого элемента порождает центр группы $B_{n}$ при $n \geqslant 3$. (Центр групшы кос был найден уже в [8], [56]. Гарсайд дал еще один способ его нахождения.)

Фундаментальная коса обладает следуюшим свойством: если элемент $b \in B_{n}^{+}$делится на $\Delta$ слева, то $b$ делится на $\Delta$ и справа. Для доказательства достаточно установить, что в моноиде $B_{n}^{+}$имеет место равенство

$$
\sigma_{i} \Delta=\Delta \sigma_{n-i} .
$$

Гарсайд доказал следуюшие два утверждения. 
ТЕОРема 5.2. Естественный гомоморфизм $B_{n}^{+} \rightarrow B_{n}$, тожддественный на образующих, $\sigma_{i} \mapsto \sigma_{i}$, является мономорфизмом.

Теорема 5.3. Каждую косу $b \in B_{n}$ можжно единственным образом представить в виде

$$
b=\Delta^{m} \cdot b^{\prime}
$$

где $b^{\prime}-$ положительная коса, не делящаяся на $\Delta$.

Гарсайд называет (10) стандартнылм видом косы $b$. Он показал, как найти представление косы в виде (10), где $b^{\prime}$ может делиться на $\Delta$. А именно, он показал, что $\Delta$ делится на каждую из образуюших, $\Delta=X_{i} \sigma_{i}$, где $X_{i} \in B_{n}^{+}$. В слове, представляющем $b$, можно сначала заменить все $\sigma_{i}^{-1}$ на $\Delta^{-1} X_{i}$, а затем вьнести все $\Delta^{-1}$ в начало слова, используя (9).

Сделав это, далее можно найти максимальную степень фундаментальной косы, на которую делится $b^{\prime}$. Для этого достаточно найти всевозможные выражения $b^{\prime}$ через образуюшие $\sigma_{i}$ (не используя $\sigma_{i}^{-1}$ ). Теорема 5.2 гарантирует, что это можно сделать за конечное время: достаточно найти все положительные слова, получающиеся из одного положительного слова, представляющего $b^{\prime}$, применением конечного числа соотношений (1). Здесь важную роль играет тот факт, что определяюшие соотношения моноида $B_{n}^{+}$сохраняют длину слова.

Алгоритм Гарсайда требует вьполнить перебор всевозможных слов, задающих коcy $b^{\prime}$, количество которых растет экспоненциально быстро с ростом длины косы. Поэтому, как и алгоритм Артина, для практического использования он не годится.

Первьй полиномиально сложный алгоритм для распознавания кос был построен Тёрстоном в [31], который существенно уточнил конструкщию Гарсайда стандартного вида косы и показал, как обойтись без экспоненциально большого перебора.

Каждой перестановке $\pi \in S_{n}$ Тёрстон сопоставил косу $s_{\pi} \in B_{n}^{+}$по следующему правилу. Перестановку $\pi$ следует разложить в произведение транспозиций $\tau_{i}=$ $(i, i+1)$ так, чтобы длина разложения была минимально возможной: $\pi=\tau_{i_{1}} \cdots \tau_{i_{m}}$. Тогда по определению $b_{\pi}=\sigma_{i_{1}} \cdots \sigma_{i_{m}}$. Нетрудно проверить, что это определение корректно. Поскольку соотношения (1) вьполнены в группе перестановок $S_{n}$, равенство $b_{\pi}=b_{\pi^{\prime}}$ имеет место, только если $\pi=\pi^{\prime}$. Множество $\left\{b_{\pi} \mid \pi \in S_{n}\right\} \subset B_{n}^{+}$ обозначим через $P_{n}$.

Элементы подмножества $P_{n} \subset B_{n}^{+}$характеризуются также тем, что представляются косами, на проекции которых все пересечения положительны и каждые две нити пересекаются не более одного раза. Эти элементы использовались и Гарсайдом, хотя он не указал на их связь с перестановками. Подмножество $P_{n}$ совпадает с множеством левых (а также с множеством правых) делителей фундаментальной косы $\Delta$ в моноиде положительных кос. Сам элемент $\Delta$ соответствует перестановке

$$
\left(\begin{array}{cccc}
1 & 2 & \ldots & n \\
n & n-1 & \ldots & 1
\end{array}\right)
$$

Тёрстон усилил теорему 5.3 следуюшим образом. 
ТеОРема 5.4. Каждая коса $b \in B_{n}$ единственным образом представляется в виде

$$
b=\Delta^{m} \cdot s_{\pi_{1}} s_{\pi_{2}} \cdots s_{\pi_{k}},
$$

где $s_{\pi_{1}} \neq \Delta, s_{\pi_{k}} \neq 1$ и для любих $i=1, \ldots, k-1, j=1, \ldots, n-1$ виполнено одно из следующих двух условий:

1) $s_{\pi_{i}}$ делится на $\sigma_{j}$ справа, т.е. $s_{\pi_{i}} \sigma_{j}^{-1} \in P_{n}$;

2) $s_{\pi_{i+1}}$ не делится на $\sigma_{j}$ слева, т.е. $\sigma_{j}^{-1} s_{\pi_{i+1}} \notin P_{n}$.

По-другому вид (11) характеризуется следуюшими образом: для каждого $i=$ $1, \ldots, k-1$ элемент $s_{\pi_{i}}$ является наибольшим обшим левым делителем (НОЛД) элементов $s_{\pi_{i}} \cdots s_{\pi_{k}}$ и $\Delta$ в моноиде $B_{n}^{+}$. Тёрстон назьвает разложение $b=$ $s_{\pi_{1}} s_{\pi_{2}} \cdots s_{\pi_{k}}$, удовлетворяюшее этому условию, жкаднылм слева видом (right-greedy form) положительной косы $b$.

У тверждение, эквивалентное теореме 5.4., было также доказано С. И. Адяном в [61].

Тёрстон указывает быстрый метод нахождения жадного слева вида данной положительной косы. Этот метод основан на следующем утверждении.

ПРЕДЛОЖЕНИЕ 5.1. Пусть $b=s_{\pi_{1}} s_{\pi_{2}} \cdots s_{\pi_{k}}-$ жадный слева вид некоторой положительной косы, $\pi \in S_{n}-$ некоторая перестановка. Тогда

$$
\text { НОЛД }\left(s_{\pi} \cdot b, \Delta\right)=\text { НОЛД }\left(s_{\pi} s_{\pi_{1}}, \Delta\right) .
$$

Кроме того, (НОЛД $\left.\left(s_{\pi} s_{\pi_{1}}, \Delta\right)\right)^{-1} s_{\pi} s_{\pi_{1}}=s_{\pi_{1}^{\prime}}$ для некоторой перестановки $\pi_{1}^{\prime}$, причем $s_{\pi_{1}^{\prime}}$ делит $s_{\pi_{1}}$ справа.

Пусть положительная коса $b$ уже приведена к жадному слева виду, $b=s_{\pi_{1}} s_{\pi_{2}} \cdots s_{\pi_{k}}$. Тогда согласно предложению 5.1 жадный слева вид косы $s_{\pi} \cdot b$ можно найти, вьполняя последовательно следующие шаги:

$$
\begin{aligned}
s_{\pi} s_{\pi_{1}} s_{\pi_{2}} \ldots s_{\pi_{k}} & \mapsto s_{\rho_{0}} s_{\pi_{1}^{\prime}} s_{\pi_{2}} \ldots s_{\pi_{k}} \\
& \mapsto s_{\rho_{0}} s_{\rho_{1}} s_{\pi_{2}^{\prime}} \ldots s_{\pi_{k}} \\
& \ldots \\
& \mapsto s_{\rho_{0}} s_{\rho_{1}} s_{\rho_{2}} \ldots s_{\rho_{k}}
\end{aligned}
$$

где $s_{\rho_{i}}=$ НОЛД $\left(s_{\pi_{i}}, s_{\pi_{i+1}}\right), s_{\pi_{i+1}^{\prime}}=s_{\rho_{i}}^{-1} s_{\pi_{i}} s_{\pi_{i+1}}, i=0,1, \ldots, k, \pi_{0}=\pi, s_{\pi_{k+1}}=1$. Это дает возможность привести произвольную положительную косу $b$ к жадному слева виду, применяя рекурсию. А именно, пусть коса $b$ представлена словом, начинаюшимся с $\sigma_{i}: b=\sigma_{i} \cdot b^{\prime}$. Поскольку $\sigma_{i} \in P_{n}$, мы получим жадньй слева вид для $b$, если сначала приведем к этому виду $b^{\prime}$, а затем воспользуемся алгоритмом выше.

Тёрстон заметил также следуюший факт. Пусть $b=s_{\pi_{1}} s_{\pi_{2}} \cdots s_{\pi_{k}}$ - некоторое разложение в произведение элементов из $P_{n}$, не обязательно каноническое. Рассмотрим новую косу $\widetilde{b}=s_{\pi_{1}^{\prime}} s_{\pi_{2}^{\prime}} \cdots s_{\pi_{k}^{\prime}}$, где $s_{\pi_{2 i+1}^{\prime}}=s_{\pi_{k-2 i}}^{-1} \cdot \Delta, s_{\pi_{2 i}^{\prime}}=\Delta \cdot s_{k-2 i+1}^{-1}$. Тогда $b=s_{\pi_{1}} s_{\pi_{2}} \cdots s_{\pi_{k}}$ имеет жадньй слева вид тогда и только тогда, когда то же верно для $\widetilde{b}=s_{\pi_{1}^{\prime}} s_{\pi_{2}^{\prime}} \cdots s_{\pi_{k}^{\prime}}$. Кроме того, в $B_{n}$ имеет место равенство $\widetilde{b}=b^{-1} \cdot \Delta^{k}$. Это дает возможность построить алгоритм для вычисления жадного слева вида косы $b \cdot s_{\pi}$, 
если $b$ уже приведена к этому виду. А именно, сначала нужно перейти к косе $s_{\pi^{\prime}} \cdot \widetilde{b}$, привести ее к жадному слева виду с помошью алгоритма вьше, а затем вьполнить операцию, обратную к $b \mapsto \widetilde{b}$.

Для произвольной косы $b \in B_{n}$ канонический вид (11) находится так. Сначала нужно представить $b$ в виде $b=\Delta^{p} \cdot b_{1}$, где $b_{1} \in B_{n}^{+}$(см. вьше). Затем косу $b_{1}$ нужно привести к жадному слева виду: $b_{1}=s_{\pi_{1}} s_{\pi_{2}} \cdots s_{\pi_{q}}$. Возможно, в этом разложении несколько первых сомножителей $s_{\pi_{1}}, s_{\pi_{2}}, \ldots$ совпадают с $\Delta$. Пусть таких сомножителей $r$ штук. Тогда каноническим видом (11) данной косы будет

$$
b=\Delta^{p+r} \cdot s_{\pi_{r+1}} \cdots s_{\pi_{q}} .
$$

Тёрстон также доказал, что для каждой косы $b$ единственно следующее разложение:

$$
b=\left(s_{\pi_{1}} s_{\pi_{2}} \cdots s_{\pi_{k}}\right)^{-1}\left(s_{\pi_{1}^{\prime}} s_{\pi_{2}^{\prime}} \cdots s_{\pi_{l}^{\prime}}\right),
$$

где $s_{\pi_{1}} s_{\pi_{2}} \cdots s_{\pi_{k}}$ и $s_{\pi_{1}^{\prime}} s_{\pi_{2}^{\prime}} \cdots s_{\pi_{l}^{\prime}}$ - жадные слева виды некоторых положительных кос такие, что НОЛД $\left(s_{\pi_{1}}, s_{\pi_{1}^{\prime}}\right)=1$ в $B_{n}^{+}$.

Для полноты картины упомянем еще два алгоритма, совершенно не похожие на перечисленные выше, которые были придуманы сравнительно недавно П. Деорнуа. Это так назьваемые алгоритм обращения слов [62] и алгоритм сокращения ручек [63]. Первый из них за квадратичное по длине исходного слова время выдает "числитель" и "знаменатель" разложения (12), не находя жадных видов для каждого из них. Второй алгоритм приводит слово, задающее косу, к виду, в котором образуюшая $\sigma_{1}$ встречается либо только в положительных, либо только в отрищательных степенях, либо не встречается вовсе. В последнем случае то же можно сказать про $\sigma_{2}$, и т. д. Деорнуа показал, что если исходное слово представляло косу $1 \in B_{n}$, то в результате работы его алгоритма получится пустое слово.

5.2. Проблема сопряженности. Проблема сопряженности в групе кос была решена почти одновременно Маканиным [29] и Гарсайдом [30], но работа Маканина не содержит полного доказательства. Мы будем следовать изложению Гарсайда.

Через $[b]$ мы будем обозначать класс сопряженности косы $b \in B_{n}$. Степень $m$ в разложении (10) Гарсайд называл степенью (power) косы $b$. Мы будем пользоваться более современной терминологией, введенной в [32], и назьвать $m$ инфимумом косы $b$ и обозначать $\inf b$. Мы имеем

$$
\inf b=\max _{\Delta^{-m} \cdot b \in B_{n}^{+}} m \text {. }
$$

Для каждого класса сопряженности $x$ Гарсайд определяет верхушку (summit) класса $x$ как

$$
\mathscr{S}(x)=\left\{b \in B_{n} \mid \inf b=\max _{b^{\prime} \in x} \inf b^{\prime}\right\}
$$

Это определение имеет смысл по следуюшей причине. Рассмотрим гомоморфизм $h: B_{n} \rightarrow \mathbb{Z}, h\left(\sigma_{i}\right)=1$ для всех $i=1, \ldots, n-1$. Будем иметь $h\left(c b c^{-1}\right)=h(b)$ для любых $b, c \in B_{n}$. Кроме того, $h(\Delta)=n(n-1) / 2$ и $h(b) \geqslant 0$ для любой положительной 
косы $b \in B_{n}^{+}$. Поэтому для любых двух кос $b, b^{\prime}$ из одного класса сопряженности вьполнено

$$
\inf b^{\prime} \leqslant \frac{2 h(b)}{n(n-1)},
$$

т.е. функция inf ограничена сверху на каждом классе сопряженности.

Мы будем использовать обозначение $\mathscr{S}(b)$ для $\mathscr{S}([b])$. Гарсайд доказал утверждение, которое в наших обозначениях формулируется так.

ПРЕДЛОЖЕНИЕ 5.2. Для любой косы $b \in B_{n}$ найдется конечная последовательность кос $s_{\pi_{i}} \in P_{n}, i=1, \ldots, N$, такая, что если положить $b_{0}=b$, $b_{i}=s_{\pi_{i}}^{-1} b_{i-1} s_{\pi_{i}}$, то будем иметь:

$$
\inf b_{0} \leqslant \inf b_{1} \leqslant \cdots \leqslant \inf b_{N}, \quad b_{N} \in \mathscr{S}(b) .
$$

Назовем сопряжение $b \mapsto s_{\pi}^{-1} b s_{\pi}$ допустимым, если inf $s_{\pi}^{-1} b s_{\pi} \geqslant \inf b$. Число допустимых сопряжений, которые можно применить к любой данной косе, конечно. Гарсайд также заметил, что для любых данных $l, m \in \mathbb{Z}$ число кос, удовлетворяюших условиям $h(b)=l$, inf $b \geqslant m$, конечно. Поэтому для любой косы $b$ можно найти все косы, получаюшиеся из $b$ конечным числом допустимых сопряжений. Отсюда вытекает следуюшее утверждение.

Теорема 5.5 (Гарсайд [30]). Существует алгоритм, который для любой косы $b \in B_{n}$, заданной некоторым словом в образующих $\sigma_{i}$, за конечное время находит $\mathscr{S}(b)$.

Это дает следуюшее решение проблемы сопряженности. Для двух данных кос $b_{1}, b_{2}$ мы можем вычислить $\mathscr{S}\left(b_{1}\right)$ и $\mathscr{S}\left(b_{2}\right)$ и затем сравнить. Результат совпадает, только если $\left[b_{1}\right]=\left[b_{2}\right]$.

Этот алгоритм Гарсайда также использует очень большой перебор. В последуюших работах [32], [64], [39], [34] он был сушественно улучшен.

Э. Аль-Рифай и Х. Мортон [32] используют, кроме инфимума, еше две характеристики косы $b$ : cynремум sup $b$ и каноническую длину $\|b\|$, которые для разложения (11) равны, соответственно

$$
\sup b=m+k, \quad\|b\|=k .
$$

Супремум характеризуется также следуюшим образом:

$$
\sup b=\min _{b^{-1} \cdot \Delta^{p} \in B_{n}^{+}} p .
$$

В [32] доказьвается следуюшее.

ПРЕДЛОЖЕНИЕ 5.3. Пусть коса $b$ имеет наименьшую каноническую длину в своем классе сопряженности, $\|b\|=\min _{b^{\prime} \in[b]}\left\|b^{\prime}\right\|$. Тогда

$$
\inf b=\max _{b^{\prime} \in[b]} \inf b^{\prime}, \quad \sup b=\min _{b^{\prime} \in[b]} \sup b^{\prime} .
$$

Таким образом, множество кос, имеюших наименьшую длину в данном классе сопряженности $x$, целиком содержится в верхушке $\mathscr{S}(x)$, определенной Гарсайдом. Из 
работы [32] видно, что использование этого подмножества позволяет построить более эффективньй алгоритм, чем алгоритм Гарсайда, поэтому имеет смысл переопределить верхушку как

$$
\mathscr{S}(x)=\left\{b \in B_{n} \mid\|b\|=\min _{b^{\prime} \in x}\left\|b^{\prime}\right\|\right\},
$$

забыв об изначальном определении Гарсайда. (Аль-Рифай и Мортон назвали подмножество (13) сверхверхушкой (super summit set), но мы предпочтем не усложнять терминологию.)

Для косы $b$, представленной в виде (11), Аль-Рифай и Мортон ввели следуюшие две операции, называемые cycling и decycling:

$$
\begin{aligned}
\mathbf{c}(b) & =\Delta^{m} \cdot s_{\pi_{2}} \cdots s_{\pi_{k}}\left(\Delta^{m} s_{\pi_{1}} \Delta^{-m}\right), \\
\mathbf{d}(b) & =\Delta^{m} \cdot\left(\Delta^{-m} s_{\pi_{k}} \Delta^{m}\right) s_{\pi_{1}} \cdots s_{\pi_{k-1}} .
\end{aligned}
$$

Обе эти операции не уменьшают inf и не увеличивают sup. Очевидно, что с $(b)$, $\mathbf{d}(b) \in[b]$. Косы $\mathbf{c}(b)$ и $\mathbf{d}(b)$ в (14) уже не имеют вида (11), однако к этому виду их можно привести с помошью указанных вьше алгоритмов Тёрстона, после чего операции $\mathbf{c}, \mathbf{d}$ можно итерировать. Так как inf не уменьшается при применении $\mathbf{c}$, последовательность $\mathbf{c}^{j}(b), j=1,2, \ldots$, не может содержать бесконечно много различных членов. Это значит, что, итерируя $\mathbf{c}$, мы рано или поздно попадем в цикл. То же верно и для операции $\mathbf{d}$. Аль-Рифай и Мортон доказали следующее.

ПРЕДЛОЖЕНИЕ 5.4. Пусть Для некоторых $j, k>0$ выполнено $b_{1}=\mathbf{c}^{j}(b)=$ $\mathbf{c}^{j+k}(b)$. Tогдa $\inf b_{1}=\max _{b^{\prime} \in[b]} \inf b^{\prime}$.

Далее, пусть для некоторых $l, m>0$ вьполнено $b_{2}=\mathbf{d}^{l}\left(b_{1}\right)=\mathbf{d}^{l+m}\left(b_{1}\right)$. Тогда $b_{2} \in \mathscr{S}(b)$.

Таким образом, чтобы найти какой-нибудь один элемент из верхушки класса [b], не обязательно использовать всевозможные сопряжения, а достаточно применять операции с и $\mathbf{d}$.

В работе Дж. Бирман, К. Ко и Дж. Ли [33] была дана следуюшая эффективная оценка для числа применений $\mathbf{c}$ и $\mathbf{d}$, достаточного для нахождения одного элемента из верхушки.

ПРЕДЛОЖЕНИЕ 5.5. Для любой косы $b \in B_{n}$ әлемент

$$
\mathbf{d}^{n(n-1) / 2-1}\left(\mathbf{c}^{n(n-1) / 2-1}(b)\right)
$$

лежит в $\mathscr{S}(b)$.

Ранее в другой статье тех же авторов [64] было проделано исследование, аналогичное работам Гарсайда и Тёрстона, но используюшее другое семейство образуюших: $a_{i j}=\left(\sigma_{i-1} \sigma_{i-2} \cdots \sigma_{j+1}\right) \sigma_{j}\left(\sigma_{i-1} \sigma_{i-2} \cdots \sigma_{j+1}\right)^{-1}, i>j$. Переход к другим образуюшим дает некоторую эффективизацию алгоритмов. Например, количество необходимых применений операций с и $\mathbf{d}$ в аналоге предложения 5.5 меняется с $n(n-1) / 2-1$ на $n-2$. 
Основньм результатом работы [33] явился полиномиальньй алгоритм для нахождения по данной косе $b$ одного элемента из верхушки класса $[b]$. Однако, для сравнения двух классов $\left[b_{1}\right]$ и $\left[b_{2}\right]$ этого недостаточно. Чтобы сравнить два класса сопряженности, нам по-прежнему нужно вычислить верхушку хотя бы одного из них полностью.

Трудность этой задачи связана с двумя обстоятельствами: во-первых, для числа элементов $\mathscr{S}(b)$ не известно оценки лучшей, чем экспоненциальная по длине косы $b$; во-вторых для того, чтобы убедиться, что найдена вся верхушка $\mathscr{S}(b)$, к каждому из найденных элементов нужно применить всевозможные допустимые сопряжения и проверить, что не получаются новые элементы.

В работе [34] показано, как обойти вторую трудность: для нахождения всей верхушки к каждому найденному элементу $a \in \mathscr{S}(b)$ достаточно применять такие допустимые сопряжения $a \mapsto s_{\pi}^{-1} a s_{\pi}$, что для любого левого собственного делителя $s_{\pi^{\prime}}$ элемента $s_{\pi} \in B_{n}^{+}$сопряжение $a \mapsto s_{\pi^{\prime}}^{-1} a s_{\pi^{\prime}}$ не является допустимьм, т.е. $s_{\pi^{\prime}}^{-1} a s_{\pi^{\prime}} \notin$ $\mathscr{S}(b)$. Оказьвается, что это сушественно сужает перебор, необходимьй для нахождения каждого следуюшего элемента из верхушки, делая его полиномиальным, и дает возможность вьполнять алгоритм на практике для кос небольшой длины (10-20 букв $\sigma_{i}$ ).

5.3. Применение к узлам. Попытка использовать подход Александера-Маркова к описанию зацеплений с помошью замкнутых кос для построения алгоритмов связана с желанием избавиться от необходимости исследовать дополнительное пространство зацепления и оперировать вместо этого объектами, имеющими "более симметричную" природу. Главная трудность с использованием кос для алгоритмической классификации зацепления связана с отсутствием простой алгебраической интерпретации второго движения Маркова (стабилизации и дестабилизации).

В работах [35]-[41] систематически разрабатьвается техника, которая, возможно, позволит добиться ясности в этом вопросе. Однако, на сегодняшний день общего алгоритма сравнения двух зацеплений, использующего специфику замкнутых кос, не построено. Имеется, однако, алгоритм для распознавания тривиального узла. Его предложили Дж. Бирман и М. Хирш в [42].

Приведем кратко идеи алгоритма Бирман-Хирша. В пространстве фиксируется цилиндрическая система координат с осью $\ell$. Классы сопряженности кос ассоциируются с замкнутыми кривыми, не пересекаюшими $\ell$ и трансверсальньми полуплоскостям $\theta=$ const, где $\theta$ - угловая координата. Если такая замкнутая коса $b$ задает тривиальньй узел, то ее можно затянуть диском $F$ таким, что $\partial F=b$. Выбрав такой диск общего положения, можно добиться, чтобы слоение $\mathscr{F}$, заданное ограничением на $F \backslash \ell$ формы $d \theta$, имело только морсовские особенности. Кроме того, от максимумов/минимумов функции $\left.\theta\right|_{F \backslash \ell}$ можно избавиться подходящей деформацией диска $F$ (см. аналогичную конструкцию ниже). Тогда слоение $\mathscr{F}$ будет иметь только седла, и без ограничения общности можно считать, что никакие два из них не лежат в одной полуплоскости $\theta=$ const.

Полученное таким образом слоение $\mathscr{F}$ можно описать комбинаторно. Добавив к комбинаторной структуре слоения $\mathscr{F}$ информацию о порядке точек пересечения $F \cap \ell$ на оси $\ell$ и циклическом порядке седел при положительном обходе вокруг $\ell$, мыполучим набор данных, по которому класс сопряженности косы $b$ восстанавливается однозначно. 
Алгоритм Бирман-Хирша делает следуюшее. Генерируется список всевозможных слоений на проколотом диске, по возрастанию сложности. Для каждого из них рассматриваются всевозможные циклические упорядочения седел и вершин (точек из $F \cap \ell)$. Для каждого полученного набора данных проверяются некоторые условия, отвечающие за их реализуемость при некотором вложении диска $D^{2}$ в $\mathbb{R}^{3}$. Затем по каждому реализуемому слоению вьписьвается коса из соответствующего класса сопряженности.

Этот алгоритм создает список представителей из всевозможных классов сопряженности кос, задаюших тривиальный узел. Для каждой данной косы $b$, представляющей тривиальный узел, ее класс сопряженности должен рано или поздно встретиться в этом списке. С помощью теории нормальных поверхностей дается оценка сверху, сколько элементов списка достаточно перебрать, чтобы в случае, если класс сопряженности косы $b$ среди них не встретился, быть уверенным, что $b$ представляет нетривиальный узел.

Строго говоря, этот алгоритм не дает нового решения проблемы алгоритмической распознаваемости тривиального узла, поскольку использует оценку, происходяшую из теорию нормальных поверхностей, которой самой по себе уже достаточно для доказательства. Однако алгоритм получается совершенно не похожим на алгоритм Хакена, и главное его преимущество состоит в том, что он генерирует список классов сопряженности кос, задающих тривиальньй узел. В работе [65] указывается, как значительно сократить необходимый для этого перебор. Основная цель этого подхода состоит в накоплении конкретной информации, которая могла бы подсказать новые идеи построения алгоритмов классификации узлов и зацеплений.

\section{6. Монотонное упрощение}

Было бы интересно найти решение проблемы сравнения узлов и зацеплений в виде алгоритма приведения к каноническому виду, принцип которого состоял бы в упрощении данного зацепления до тех пор, пока это возможно, так, чтобы полученное в результате упрощения представление данного зацепления определялось его изотопическим типом. В частности, любую диаграмму тривиального узла такой алгоритм должен монотонно распутывать до некоторой стандартной, простейшей, диаграммы. (Заметим, что подобный подход наиболее близок к принщипу, которым мы пользуемся, распутьвая запутанную веревку.)

Известно много примеров плоских диаграмм тривиального узла, из которых нельзя получить диаграмму без пересечений с помошью движений Райдемайстера, не увеличивающих сложность диаграммы по дороге. Бесконечная серия таких примеров построена в [66]. Простейшая диаграмма из этой серии показана на рис. 10.

Подход Александера-Маркова также не дает алгоритма монотонного упрошения. В [67] показано, что замыкание косы $\beta=\sigma_{3}^{-1} \sigma_{2} \sigma_{3}^{-1} \sigma_{2} \sigma_{1}^{3} \sigma_{2}^{-1} \sigma_{1} \sigma_{2}^{-1} \in B_{4}$ является тривиальным узлом, однако любая последовательность движений Маркова, преврашаюшая $\beta$ в косу $1 \in B_{1}$, должна использовать стабилизацию. Это значит, что количество нитей, а значит, и сложность косы, должны быть в какой-то момент увеличены.

Следующее утверждение, доказанное автором недавно [43], показьвает, что идея приведения зацеплений к каноническому виду имеет право на существование. 


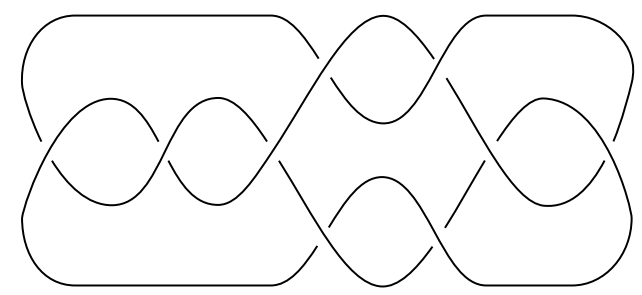

Рис. 10. Диаграмма тривиального узла, которую нельзя монотонно упростить движениями Райдемайстера

ТЕОРема 6.1. Любая прямоугольная диаграмма тривиального узла допускает нестрого монотонное упрощение әлементарными преобразованиями, т.е. может быть превращена в простейшую диаграмму (сложности два) ииклическими перестановками, рокировками и дестабилизациями.

Определение прямоугольных диаграмм и элементарных преобразований см. в разделе 2. Для доказательства теоремы 6.1 используются так называемые книжные представления зацеплений (arc-presentations of links), cм. определение ниже. Прямоугольные диаграммы - это способ кодирования книжных представлений. Поэтому фактически теорема 6.1 утверждает возможность монотонного упрощения любого книжного представления тривиального узла.

Мы приведем здесь полное доказательство теоремы 6.1, несколько отличное от того, которое дано в [43]. Идеи доказательства во многом заимствованы автором из работ [68], [39], [51], поэтому сначала мы дадим подробную историческую справку.

В [68] Д. Беннекен доказал следуюшее неравенство, связьвающее число нитей $n$ произвольной косы $b$, алгебраическое число $m$ пересечений в $b$ и наименьший возможньй род $g$ поверхности Зейферта для $L_{b}$, ориентация которой согласована с ориентацией зацепления $L$ :

$$
2 g-1 \geqslant|m|-n .
$$

Идея доказательства состояла в следующем. Беннекен рассмотрел в $S^{3}$ слоение Риба $\mathscr{R}$ и представил замкнутые косы как зацепления, трансверсальные этому слоению. Напомним, что слоение Риба содержит один замкнутый слой, гомеоморфньй двумерному тору $\mathbb{T}^{2}$, который разделяет $S^{3}$ на два полнотория, назовем их $M_{1}$ и $M_{2}$. Любое зацепление, трансверсальное $\mathscr{R}$, должно целиком лежать в $M_{1}$ или $M_{2}$ и иметь вид замкнутой косы. Будем считать, что оно лежит в $M_{1}$.

Далее Беннекен рассмотрел ограничение $\mathscr{F}$ слоения $\mathscr{R}$ на произвольную поверхность Зейферта $F, \partial F=L_{b}$. Он потребовал, чтобы пересечение $F \cap M_{2}$ состояло из меридианальных дисков. Беннекен показал, что заменой пары $L_{b}, F$ на изотопную $L_{b^{\prime}}, F^{\prime}$ можно избавиться от наличия в слоении $\mathscr{F}$ определенных фрагментов. Для слоения, не содержащего этих фрагментов, неравенство Беннекена получается элементарным подсчетом эйлеровой характеристики $\chi(F)$ в терминах особенностей слоения $\mathscr{F}$.

Нам не понадобится неравенство Беннекена, но понадобятся топологические приемы, которыми он заменяет пару зацепление+поверхность на изотопную так, чтобы слоение $\mathscr{F}$ изменялось заранее предписанным образом. Мы назьваем эти приемы трюками Беннекена. Важная особенность этих приемов состоит в том, что возможность 
их применения гарантируется структурой слоения $\mathscr{F}$. Это значит, что, не глядя, как именно поверхность $F$ вложена в $S^{3}$, а зная только, как вьглядит на ней слоение $\mathscr{F}$, мы можем сказать, какие из трюков Беннекена заведомо можно применить и как будет вьглядеть слоение после этого.

Заметим, что роль слоения внутри полнотория $M_{2}$ в конструкции Беннекена несущественна, поэтому слоение Риба можно заменить на более простое: полноторие $M_{2}$ можно стянуть в окружность $S^{1} \subset S^{3}$, дополнение к которой будет расслоено открытыми дисками такими, что $S^{1}$ будет краем замыкания каждого из них.

Заметим также, что Беннекен не обсуждает вопрос, как на алгебраическом языке описать изменение косы $b \mapsto b^{\prime}$ в результате его трюков, поскольку преследует лишш цель доказать неравенство (15).

В серии работ [35]-[40] Бирман и Менэско получили ряд результатов с использованием трюков Беннекена. Они рассматривали расслоение $S^{3} \backslash S^{1}$ на диски и представляли замкнутые косы как зацепления, трансверсальные этому слоению. Бирман и Менэско проанализировали, что происходит с косой в результате применения трюков Беннекена и ввели операцию обмена (exchange move), показанную на рис. 11. Операция обмена разлагается в композицию движений Маркова, включающих одну стабилизацию, одну дестабилизацию и сопряжения:

$$
\begin{aligned}
\sigma_{n}^{-1} b \sigma_{n} b^{\prime} & \mapsto \sigma_{n}^{-2} b \sigma_{n} b^{\prime} \sigma_{n} \mapsto \sigma_{n}^{-2} b \sigma_{n} b^{\prime} \sigma_{n} \sigma_{n+1} \\
& \mapsto \sigma_{n} \sigma_{n+1} \sigma_{n}^{-2} b \sigma_{n} b^{\prime}=\sigma_{n+1}^{-2} \sigma_{n} \sigma_{n+1} b \sigma_{n} b^{\prime} \mapsto \sigma_{n} b \sigma_{n+1} \sigma_{n} \sigma_{n+1}^{-2} b^{\prime} \\
& \mapsto \sigma_{n} b \sigma_{n}^{-2} \sigma_{n+1} \sigma_{n} b^{\prime} \mapsto \sigma_{n} b^{\prime} \sigma_{n} b \sigma_{n}^{-2} \sigma_{n+1} \mapsto \sigma_{n} b^{\prime} \sigma_{n} b \sigma_{n}^{-2} \mapsto \sigma_{n} b \sigma_{n}^{-1} b^{\prime}
\end{aligned}
$$
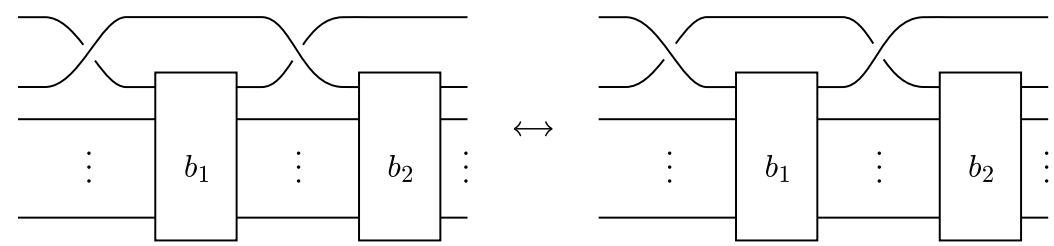

Рис. 11. Операция обмена для кос

Бирман и Менэско даказали следуюшие три утверждения.

ТеОрема 6.2. Если $L_{b}-$ тривиальный узел, то из б можно получить $1 \in B_{1}$ конечным числом операчий обмена, сопряжений и дестабилизачий.

Теорема 6.3. Если $L_{b}$ - разводимое зачепление, то конечным числом операций обмена и сопряжений $b$ можно привести $\kappa$ виду $b=b_{1} b_{2}$, где $b_{1} \in\left\langle\sigma_{1}, \ldots\right.$, $\left.\sigma_{i-1}\right\rangle, b_{2} \in\left\langle\sigma_{i+1}, \ldots, \sigma_{n-1}\right\rangle$.

Теорема 6.4. Если $L_{b}$ - составное зацепление, то конечным числом операчий обмена и сопряжений $b$ можно привести $\kappa$ виду $b=b_{1} b_{2}$, где $b_{1} \in\left\langle\sigma_{1}, \ldots\right.$, $\left.\sigma_{i-1}\right\rangle, b_{2} \in\left\langle\sigma_{i}, \ldots, \sigma_{n-1}\right\rangle$. 
ЗАмЕчАНИЕ. В доказательстве последней теоремы имеется пробел, который был указан в [43] и, как затем показали Бирман и Менэско, может быть легко устранен (исправление должно появиться в печати в ближайшее время). Указанные результаты Бирман и Менэско можно также получить как следствия аналогичных утверждений для книжных представлений [43], что неудивительно, поскольку для доказательства последних использован похожий метод.

ЗАмЕчАНИЕ. В своих работах Бирман и Менэско использовали более общую операцию обмена, в которой вместо каждой из нитей, показанной на рис. 11, может быть несколько параллельных. Такая, более общая, операция разлагается в композицию обычных операций обмена и сопряжений. Этот факт не указан явно в упомянутых работах, хотя доказательство его несложно и безусловно известно их авторам.

Неформально говоря, результаты Бирман-Менэско можно сформулировать так: для того чтобы найти полное разложение данного зацепления, представленного в виде замькания косы, на простые неразводимые слагаемые и тривиальные узлы, достаточно применять движения Маркова и операции обмена, не увеличивая количество нитей косы. Однако из этого утверждения не следует немедленно существование алгоритма разложения, основанного на простом переборе кос, получаемых указанньми операциями, поскольку группа кос $B_{n}$ при $n \geqslant 2$ бесконечна.

Оказалось, что разработанньй на основе трюков Беннекена метод Бирман-Менэско расслоенных поверхностей работает столь же эффективно для книжных представлений. Это было замечено П. Кромвеллом [51], которьй, однако, не поставил вопроса о монотонном упрошении с помошью элементарных преобразований, а показал лишш, что сложность зацепления, измеряемая как минимально возможная сложность его книжного представления, аддитивна (после вычитания двух) при взятии операции связной суммы. Иначе говоря, взяв связную сумму двух книжных зацеплений, простейших в своих классах, некоторым стандартным образом, мы получим книжное представление, которое будет иметь наименьшую возможную сложность в своем классе эквивалентности. При этом Кромвелл повторил ошибку, сделанную Бирман и Менэско в [38].

Хотя Кромвелл и не обсуждает вопроса о монотонном упрощении книжных представлений зацеплений, к содержащимся в его работе аргументам достаточно добавить лишь несколько технических замечаний, чтобы доказать следуюшее утверждение.

ТЕОРема 6.5. Если прямоугольная диаграмма $R$ задает разводимое зачепление, то за конечное число рокировок и чиклических перестановок ее можсн превратить в несвязную сумму двух нетривиальных диаграмм, т.е. прямоугольную диаграмму, содержашую только вериинь $(i, j)$, для которых $i, j \leqslant k$ или $i, j \geqslant k+1$, где $k$ - некоторое целое в интервале $[2, c(R)-1]$.

В работе Кромвелла содержится также сушественная часть доказательства аналогичной теоремы для связных сумм.

ТЕОРема 6.6. Если прямоугольная диаграмма $R$ задает составное неразводимое зачепление, то за конечное число рокировок и ииклических перестановок ее можсно превратить в связную сумму двух нетривиальных диаграмм, т.е. прямоугольную диаграмму, все вершины $(i, j)$ которой, кроме двух, удовлетворяют $i, j \leqslant k$ или $i, j \geqslant k+1$, где $k$ - некоторое челое в интервале $[2, c(R)-1]$. 
Для доказательства нужны те же технические замечания, что и для предыдущей теоремы, и нужно рассмотреть случай слоения, пропушенный в [51], а также в [38].

Возможность применения метода Бирман-Менэско для книжных представлений тривиального узла Кромвелл не рассматривал.

Теоремы 6.5 и 6.6 означают сушествование алгоритма для полного разложения зацепления на простые неразводимые слагаемые и тривиальные узлы. Для нахождения этого разложения нужно представить данное зацепление некоторой прямоугольной диаграммой $R$ и затем перебрать все прямоугольные диаграммы, которые можно получить из $R$ элементарными преобразованиями, не включаюшими стабилизацию. Как мы знаем (см. предложение 2.1), этот перебор конечен.

Теперь мы приступим к доказательству теоремы 6.1.

В пространстве $\mathbb{R}^{3}=S^{3} \backslash \infty$ зафиксируем некоторую ориентированную прямую $\ell$ и свяжем с ней цилиндрическую систему координат $(x, r, \theta)$, где $x$ - координата на $\ell$, а $\theta$ - угловая координата (координату $r$ мы не будем использовать). Замыкания полу-

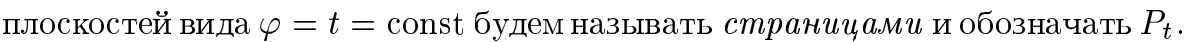

Книжнылм представлением данного изотопического класса зацеплений $X$ (или, просто, книжсным зацеплением из $X$ ) называется зацепление $L \in X$ такое, что

1) пересечение $L \cap \ell$ состоит из конечного числа точек, которые мы называем вершинами;

2) $L$ состоит из конечного числа простых дуг, каждая из которых лежит в некоторой странище $P_{t}$;

$3)$ каждая странища $P_{t}$ содержит не более одной дуги, входящей в $L$.

Заметим, что зацепления $L_{R}$, построенные в разделе 2 (см. (2)), являются книжными зацеплениями по отношению к обеим прямым $x=z=0$ и $y=0, z=1$. В дальнейшем нам будет удобно считать, что дуги $L \cap P_{t}$ гладкие.

Соответствие между книжньми представлениями зацеплений и прямоугольными диаграммами устанавливается следуюшим образом. Геометрическая прямоугольная диаграмма, соответствующая книжному зацеплению $L$, имеет вершинами все точки $(x, y)$ такие, что $y \in[0,2 \pi)$ и $L$ содержит дугу в $P_{y}$, один из конщов которой $x \in \ell \cong \mathbb{R}$. Чтобы получить "алгебраическую" прямоугольную диаграмму, соответствующую $L$, нужно занумеровать дуги $L \cap P_{t}$ по возрастанию координаты $t$ и вершины $L \cap \ell$ по возрастанию $x$, а затем вьписать таблицу инциденций между вершинами и дугами зацепления $L$.

При деформации зацепления в классе книжных зацеплений с фиксированным числом вершин соответствуюшая прямоугольная диаграмма может претерпевать только циклические перестановки горизонтальных ребер. Мы будем рассматривать ось $\ell$ как замкнутую окружность, причисляя к ней точку $\infty$, а страницы $P_{t}-$ как замкнутые двумерные диски. Тогда деформация книжного зацепления может также вызьвать и циклические перестановки вертикальных ребер. Таким образом, комбинаторные классы книжных представлений - это прямоугольные диаграммы, рассматриваемые с точностью до циклических перестановок.

Введем некоторые обозначения и определения. Для трех точек $x_{1}, x_{2}, x_{3}$ ориентированной окружности будем писать $x_{2} \in\left(x_{1}, x_{3}\right)$, если точки $x_{1}, x_{2}, x_{3}$ попарно различны и при положительном обходе вдоль $S^{1}$ встречаются в таком порядке: $\ldots, x_{1}, x_{2}, x_{3}, x_{1}, x_{2}, \ldots$ Мы также пишем $x_{2} \in\left[x_{1}, x_{3}\right]$, если $x_{2} \in\left(x_{1}, x_{3}\right)$ или 
$x_{2} \in\left\{x_{1}, x_{3}\right\}$. Через $\gamma_{t, x_{1}, x_{2}}$ мы будем обозначать любую дугу, собственно вложенную в $P_{t}$, с концами $\partial \gamma_{t, x_{1}, x_{2}}=\left\{x_{1}, x_{2}\right\}$.

Дуги $\gamma_{t_{1}, x_{1}, x_{1}^{\prime}}, \gamma_{t_{2}, x_{2}, x_{2}^{\prime}}$, входящие в некоторое книжное зацепление $L$, назьваются соседними в $L$, если $L$ не имеет дуг в страницах $P_{t}$ при $t \in\left(t_{1}, t_{2}\right)$ или при $t \in\left(t_{2}, t_{1}\right)$. Соседние дуги $\gamma_{t_{1}, x_{1}, x_{1}^{\prime}}, \gamma_{t_{2}, x_{2}, x_{2}^{\prime}}$ назьваются скрещивающимися, если пары $\left(x_{1}, x_{1}^{\prime}\right)$ и $\left(x_{2}, x_{2}^{\prime}\right)$ зацеплены. Соседние вершины $x_{1}, x_{2}$ назьваются скрещивающимися, если зацеплены пары $\left(t_{1}, t_{1}^{\prime}\right)$ и $\left(t_{2}, t_{2}^{\prime}\right)$, где $t_{i}, t_{i}^{\prime}$ - это $\theta$-координаты дуг, концом которых является $x_{i}, i=1,2$.

Нам понадобятся следуюшие операции над книжными зацеплениями, которые не меняют их изотопического типа (они введены также в [51]):

перестановка соседних нескрещивающихся дуг: соседние нескрещивающиеся дуги $\gamma_{t_{1}, x_{1}, x_{1}^{\prime}}, \gamma_{t_{2}, x_{2}, x_{2}^{\prime}}$ заменяются на $\gamma_{t_{2}, x_{1}, x_{1}^{\prime}}, \gamma_{t_{1}, x_{2}, x_{2}^{\prime}}$;

перестановка соседних нескрещивающихся вершин: для двух нескрешивающихся вершин $x_{1}, x_{2}$ дуги $\gamma_{t_{1}^{\prime}, x_{1}, x_{1}^{\prime}}, \gamma_{t_{1}^{\prime \prime}, x_{1}, x_{1}^{\prime \prime}}, \gamma_{t_{2}^{\prime}, x_{2}, x_{2}^{\prime}}, \gamma_{t_{2}^{\prime \prime}, x_{2}, x_{2}^{\prime \prime}}$ заменяются на $\gamma_{t_{1}^{\prime}, x_{2}, x_{1}^{\prime}}, \gamma_{t_{1}^{\prime \prime}, x_{2}, x_{1}^{\prime \prime}}, \gamma_{t_{2}^{\prime}, x_{1}, x_{2}^{\prime}}, \gamma_{t_{2}^{\prime \prime}, x_{1}, x_{2}^{\prime \prime}}$

стабилизация: замена некоторой дуги $\gamma_{t, x_{1}, x_{2}} \subset L$ на пару соседних дуг $\gamma_{t, x_{1}, x_{3}} \cup \gamma_{t+\varepsilon, x_{2}, x_{3}}$, где $x_{3} \notin L$;

дестабилизация: операция, обратная к стабилизации.

В терминах соответствуюших прямоугольных диаграмм перестановки нескрещиваюшихся дуг и нескрешиваюшихся вершин соответствуют рокировкам. Введенная нами операция стабилизации для прямоугольных диаграмм представляет собой частный случай стабилизации для книжных зацеплений. Общий случай стибилизации книжных зацеплений с помошью рокировок сводится к частному, когда добавляемая вершина $x_{3}$ является соседней с $x_{1}$. Это проиллюстрировано на рис. 12 с помошью соответствуюших прямоугольных диаграмм.

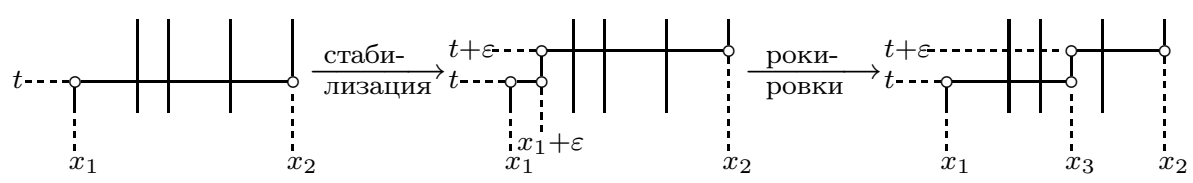

Рис. 12. Сведение общего случая стабилизации к частному

Нам также понадобится следующая операция, обобщающая перестановку вершин, которую мы называем обобщенной рокировкой. Пусть $x_{1}, x_{2}, x_{3} \in \ell \backslash L, x_{2} \in\left(x_{1}, x_{3}\right)$, $t_{1}, t_{2}, t_{3} \in S^{1}=\mathbb{R} /(2 \pi \mathbb{Z})$ таковы, что

1) ни одна дуга $\gamma_{t, x, x^{\prime}} \subset L$ не пересекается с $\gamma_{t, x_{1}, x_{2}}$ при $t \in\left[t_{1}, t_{2}\right]$;

2) ни одна дуга $\gamma_{t, x, x^{\prime}} \subset L$ не пересекается с $\gamma_{t, x_{2}, x_{3}}$ при $t \in\left[t_{2}, t_{1}\right]$.

Тогда мы назьваем обобшенной рокировкой перестановку всех вершин из $\left(x_{1}, x_{2}\right) \mathrm{c}$ вершинами из $\left(x_{2}, x_{3}\right)$ с сохранением порядка вершин внутри каждого из интервалов. Опишем эту операцию более формально. Без ограничения обшности можем считать $x_{1}, x_{3} \neq \infty, x_{1}<x_{2}<x_{3}$. Рассмотрим функщию

$$
f(x)= \begin{cases}x+x_{3}-x_{2}, & \text { если } x \in\left(x_{1}, x_{2}\right), \\ x+x_{1}-x_{2}, & \text { если } x \in\left(x_{2}, x_{3}\right), \\ x & \text { в противном случае. }\end{cases}
$$


Определяемая нами обобшенная рокировка заменяет в $L$ каждую дугу вида $\gamma_{t, x, x^{\prime}}$ на $\gamma_{t, f(x), f\left(x^{\prime}\right)}$

На рис. 13 продемонстрировано, что обобщенная рокировка раскладывается в композицию перестановок дуг и перестановок вершин.
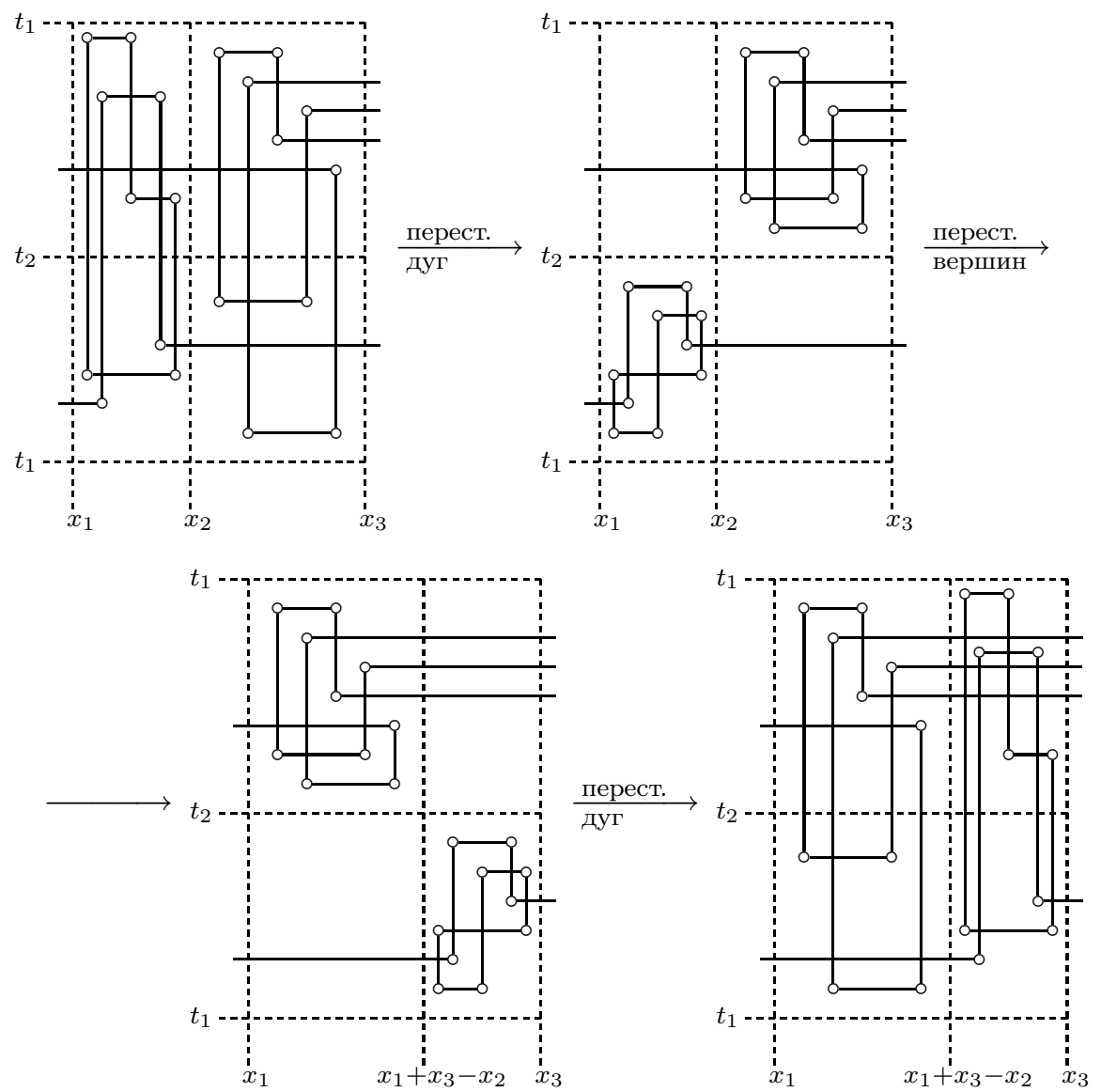

Рис. 13. Разложение обобщенной рокировки в композицию обычных

Пусть $K$ - книжное представление тривиального узла. Тогда найдется вложенньй двумерный диск $F \subset S^{3}, F \cong D^{2}$, границей которого является $K$. Этот диск можно выбрать так, чтобы он удовлетворял следующим условиям общего положения:

- поверхность $F$ гладкая вплоть до края (за исключением вершин узла $K$, где нарушена гладкость самого узла);

- внутренность диска $F$ пересекает ось $\ell$ трансверсально в конечном числе точек;

- ограничение (многозначной) функции $\theta$ на $F \backslash \ell$ имеет критические точки только морсовского типа, т.е. седла, максимумы и минимумы.

Рассмотрим слоение с особенностями $\mathscr{F}$ на диске $F$, заданное уравнением $d \theta=0$. Иначе говоря, слоями $\mathscr{F}$ являются компоненты пересечений $F \cap P_{t}$. Заметим, что поверхность $F$ может касаться странищ $P_{t}$ и в точках края. В этом случае слоение $\mathscr{F}$ 
будет иметь особенность типа седла на крае. В точках $F \cap \ell$ форма $d \theta$ не определена вовсе. Мы будем назьвать точки $F \cap \ell$ вершинами слоения $\mathscr{F}$, различая внутренние, т.е. лежашие во внутренности диска $F$, и граничные, которые являются вершинами узла $K$. Поведение слоения $\mathscr{F}$ вблизи внутренних и граничных вершин показано на рис. 14 а) и б) соответственно. Особенности слоения $\mathscr{F}$, показанные на рис. 14 в), г) и д), мы назьваем полюсами, внутренними седлами и граничными седлами соответственно.

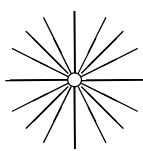

a)

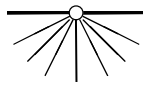

б)

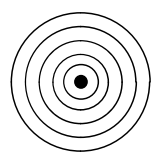

B)

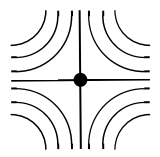

г)

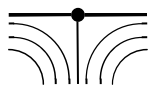

д)

Рис. 14. Слоение $\mathscr{F}$ возле вершин и особых точек

Наложим дополнительное условие на поверхность $F$ :

- никакие два седла, хотя бы одно из которых внутреннее, не соединены сепаратрисой.

Вьполнения этого ограничения всегда можно добиться шевелением поверхности $F$. Отметим, что наличие нескольких седел на одной дуге вида $K \cap P_{t}$ не запрещено, поскольку мальм шевелением поверхности $F$ при фиксированном крае такая ситуация не устраняется.

Диск $F$ можно продеформировать так, что слоение $\mathscr{F}$ не будет иметь полюсов и замкнутых слоев. Делается это точно так же, как, например, в [39]: сначала из диска $F$ удаляются все замкнутые слои, полюсы и особые слои, имеюшие вид "восьмерки" (т.е. такие, в окрестности которых лежат лишш замкнутые неособые слои). Получается диск с дырками, край каждой из которых лежит в некоторой странище $P_{t}$ и содержит одно внутреннее седло. На рис. 15 слева показано, как вьглядит слоение в окрестности новой компоненты края. После добавления к нашей поверхности плоского диска, ограничиваемого этой кривой, и малого шевеления, получаем слоение, показанное на рис. 15 справа.
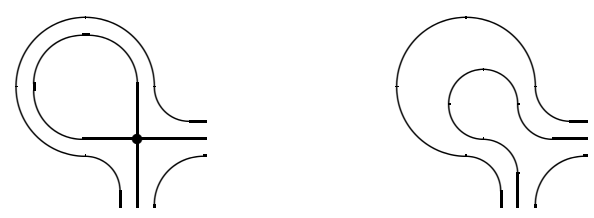

Рис. 15. Удаление замкнутых слоев

Далее мы будем работать с дисками $F$, для которых слоение $\mathscr{F}$ не имеет замкнутых слоев и полюсов. Неособые слои $\mathscr{F}$ - это открытые дуги, соединяющие две вершины слоения $\mathscr{F}$. Замыкание объединения неособых слоев, подходящих к данной вершине $v$, будем называть звездой вершины $v$. Количество седел (внутренних и граничных), содержашихся в звезде вершины $v$, назовем валентностью вершины $v$. 
a)

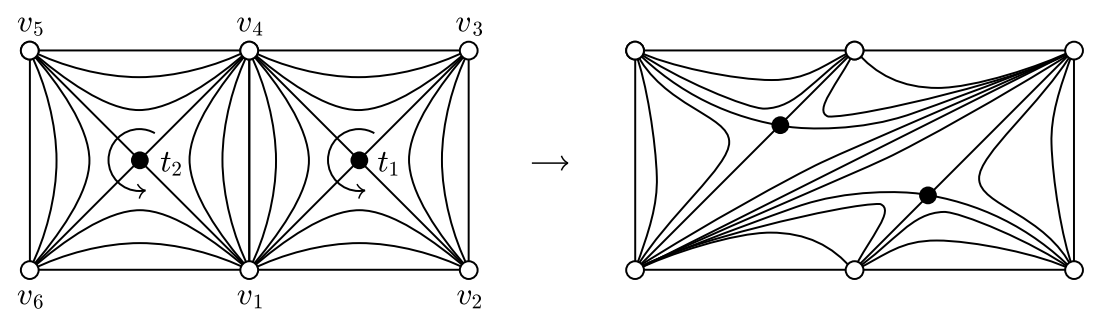

б)

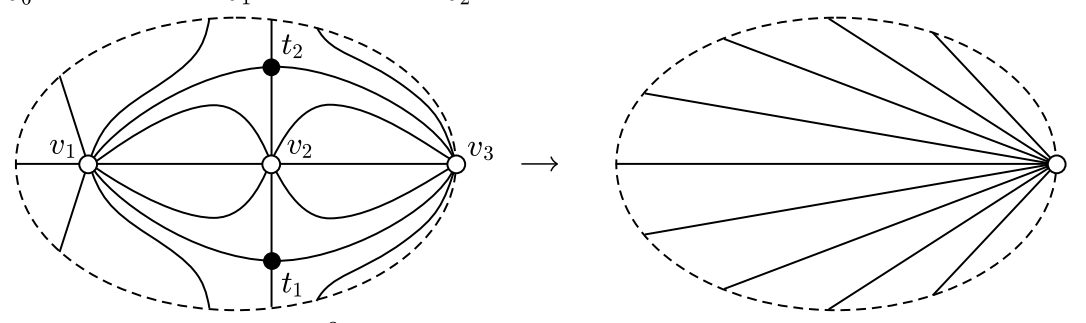

в)
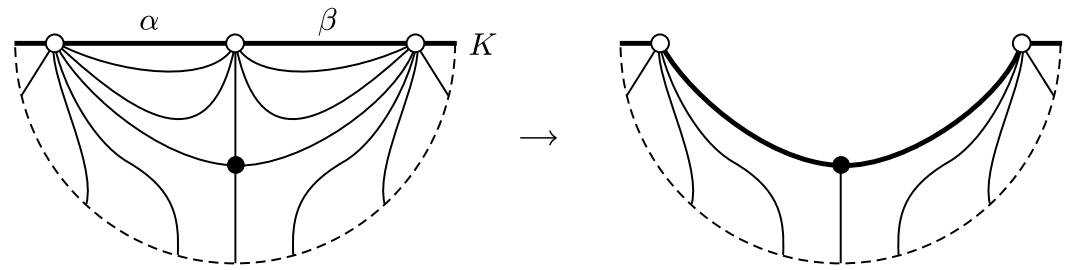

г)
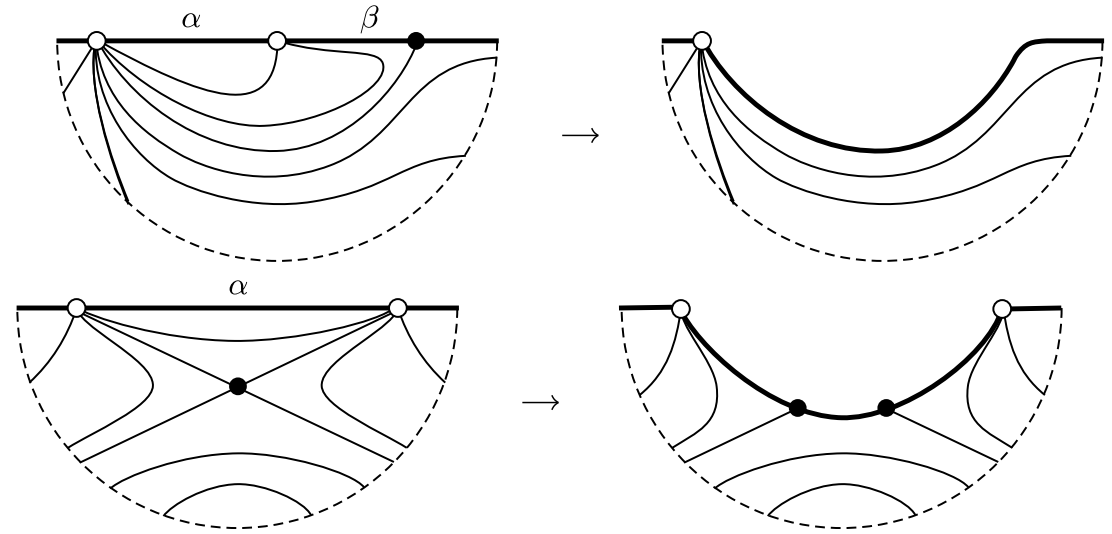

e)
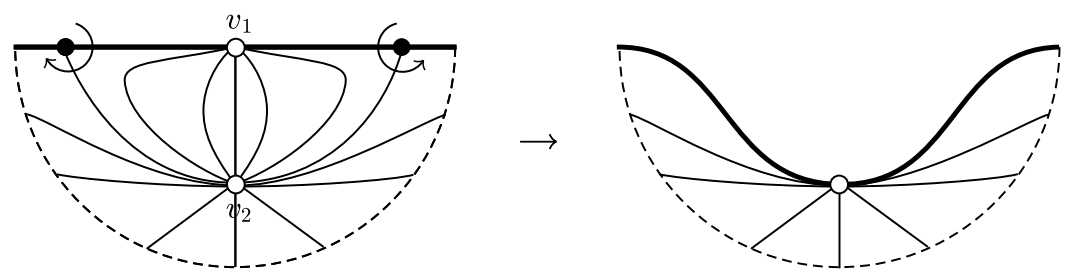

Ж)
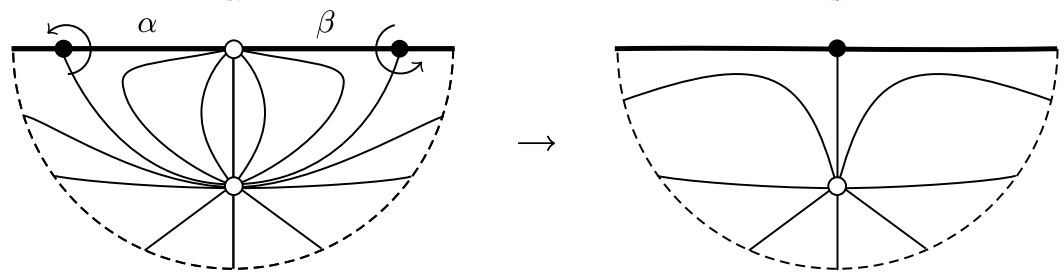

Рис. 16. Упрощение слоения $\mathscr{F}$ 
Внутреннюю вершину назовем хорошей, если в ее звезде не более одной вершины является граничной. Граничную вершину $v$ назовем хорошей, если в границе ее звезды нет граничных вершин, кроме тех, с которыми $v$ соединена отрезком дуги, содержашейся в $K=\partial F$.

Обозначим через $V_{k}$ количество внутренних вершин валентности $k$, через $V_{k}^{\mathrm{b}}-$ количество граничных вершин валентности $k$, через $s$ - число внутренних седел, через $s^{\mathrm{b}}$ - число граничных седел и, наконец, через $q$ - количество дуг в $K=\partial F$, содержащих хотя бы одно граничное седло. Мы имеем

$$
\begin{aligned}
& c(K)=\sum_{k} V_{k}^{\mathrm{b}}, \\
& 2 q \leqslant c(K)+s^{\mathrm{b}} .
\end{aligned}
$$

Введем понятие сложности диска $F$ как следующую четверку целых чисел:

$$
c(F)=\left(c(K), s, s^{\mathrm{b}}, V_{3}^{\prime}\right),
$$

где $V_{3}^{\prime}$ - количество хороших трехвалентных вершин. Для таких четверок введем лексикографический порядок: $(i, j, k, l)<\left(i^{\prime}, j^{\prime}, k^{\prime}, l^{\prime}\right)$, если либо $i<i^{\prime}$, либо $i=i^{\prime}$ и $j<j^{\prime}$, и т. д.

Для каждого седла $x$ мы определим ориентацию, которая задается циклическим порядком вершин, соединенных с $x$ сепаратрисами, на оси $\ell$.

Теперь мы введем набор операций, которыми диск $F$ можно монотонно упростить. Обратимся к рис. 16 а)-ж). Мы утверждаем, что для каждого из них вьполнено следующее:

если в слоении $\mathscr{F}$ встретился фрагмент, изображенньй на рисунке слева, то найдется другой диск $F^{\prime}$, граница которого $K^{\prime}=\partial F^{\prime}$ также является книжньм узлом, такой, что: 1$)$ слоение $\mathscr{F}^{\prime}$ на $F^{\prime}$ получается из слоения $\mathscr{F}$ заменой левого фрагмента на правый; 2) узел $K^{\prime}$ получается из $K$ конечным числом рокировок и (возможно) дестабилизацией.

Отложив на время доказательство этого факта, проверим, что этими операциями (а также им симметричньми) диск $F$ всегда можно упростить до диска, показанного на рис. 17.

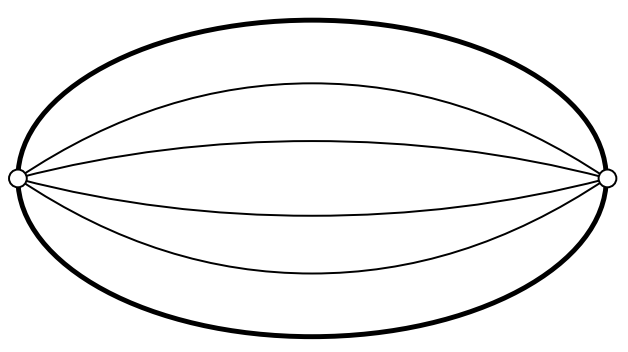

Рис. 17. Простейшее слоение $\mathscr{F}$ 
Если слоение $\mathscr{F}$ имеет хорошую одновалентную граничную вершину, то слоение упрощается одной из операций в), г). При этом упрощается узел $K=\partial F$.

Если слоение $\mathscr{F}$ имеет хорошую двухвалентную внутреннюю вершину, то его можно упростить операцией б). Если таких вершин нет, но есть хорошая трехвалентная внутренняя вершина, то два из трех седел, входяших в ее звезду, имеют одинаковую ориентацию, и слоение можно упростить операцией а). При этом хорошая трехвалентная вершина $v_{1}$ преврашается в хорошую двухвалентную и число хороших трехвалентных вершин уменьшается.

Если на какой-то из дуг, составляющих $K$, нет ни одного граничного седла и хотя бы один из ее концов является хорошей граничной вершиной, то можно применить операцию д). При этом уменьшится число внутренних седел.

Наконец, если таких дуг нет, но имеется хорошая двухвалентная граничная вершина, то слоение упрощается операцией е) или ж). В случае е) уменьшается число граничных седел, в случае ж) упрощается узел $K$.

Таким образом, для того, чтобы слоение $\mathscr{F}$ можно было упростить одной из операций, показанных на рис. 16 , достаточно наличия в нем хорошей внутренней вершины валентности $\leqslant 3$ или хорошей граничной вершины валентности $\leqslant 2$.

Из выражения эйлеровой характеристики через топологические индексы особенностей имеем

$$
1=\sum_{k} V_{k}+\frac{1}{2} \sum_{k} V_{k}^{\mathrm{b}}-s-\frac{1}{2} s^{\mathrm{b}} .
$$

Подсчитав сепаратрисы двумя разными способами, получаем

$$
\sum_{k} k\left(V_{k}+V_{k}^{\mathrm{b}}\right)=4 s+s^{\mathrm{b}}+2 q .
$$

Из (16), (19), (20) получаем

$$
4=\sum_{k}(4-k) V_{k}+\sum_{k}(3-k) V_{k}^{\mathrm{b}}-c(K)-s^{\mathrm{b}}+2 q .
$$

Заметим, что внутренних вершин валентности $\leqslant 1$ и граничных вершин валентности нуль в слоении $\mathscr{F}$ нет, если оно не имеет вида, показанного на рис. 17. Поэтому (21), с учетом (17), дает

$$
2 V_{2}+V_{3}+2 V_{1}^{\mathrm{b}}+V_{2}^{\mathrm{b}}=4+\sum_{k \geqslant 5}(k-4) V_{k}+\sum_{k \geqslant 4}(k-3) V_{k}^{\mathrm{b}}+c(K)+s^{\mathrm{b}}-2 q \geqslant 4
$$

Если все вершины являются хорошими, то из последнего неравенства следует возможность применить одну из операший на рис. 16. Предположим, что хороших внутренних вершин валентности $\leqslant 3$ и хороших граничных вершин валентности $\leqslant 2$ нет. Разрежем диск $F$ вдоль регулярных слоев слоения $\mathscr{F}$, соединяюших плохие внутренние вершины с граничньпи, и слоев, соединяюших пары плохих граничных вершин, см. рис. 18. Пусть Г - объединение всех разрезов.

Среди дисков, полученных в результате разрезания, будет по меньшей мере два концевых диска, т.е. таких, граница которых соприкасается с Г только один раз. На рис. 18 мы пометили конщевые диски знаком $*$. 


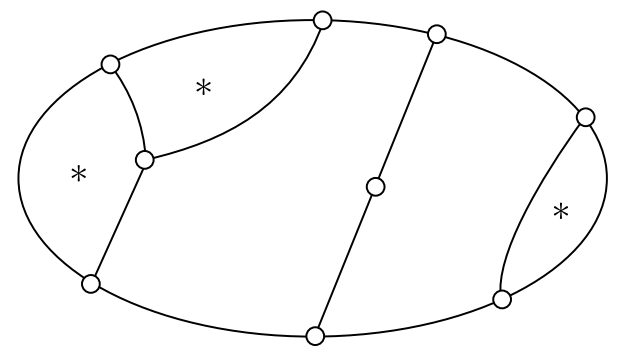

Рис. 18. Разрезание диска F по "перетяжкам"

Для конщевого диска $d$ можно повторить вычисления, сделанные вьше, и получить:

$$
2 V_{2}+V_{3}+2 V_{1}^{\mathrm{b}}+V_{2}^{\mathrm{b}} \geqslant 4+c+s^{\mathrm{b}}-2 q
$$

где все обозначения $V_{2}, V_{3}, s^{\mathrm{b}}, \ldots$ относятся к количеству вершин, седел и т. д. в $d$. Так как во всем диске $F$ не было внутренних хороших вершин валентности $\leqslant 3$, их нет и в $d$. Плохих вершин в $d$ нет по построению. Так что для диска $d$ имеем

$$
2 V_{1}^{\mathrm{b}}+V_{2}^{\mathrm{b}} \geqslant 4+c+s^{\mathrm{b}}-2 q
$$

Хорошие одно- и двухвалентные вершины в $\partial d$ могут быть только в $\partial d \cap \Gamma$. Возможны следуюшие два случая.

Случай 1: пересечение $\partial d \cap \Gamma$ состоит из одной дуги. Коншы дуги $\partial d \cap \Gamma$ не могут иметь валентность 1 , поэтому имеем $V_{2}^{\mathrm{b}} \leqslant 2, V_{1}^{\mathrm{b}}=0$. Это противоречит неравенству (23).

Случай 2: пересечение $\partial d \cap \Gamma$ состоит из двух дуг. Так как на этих дугах нет граничных седел, имеем $c+s^{\mathrm{b}}-2 q \geqslant 2$. Из трех вершин, входяших в $\partial d \cap \Gamma$, не более двух имеют валентность 1 . Отсюда $V_{1}^{\mathrm{b}} \leqslant 2, V_{1}^{\mathrm{b}}+V_{2}^{\mathrm{b}} \leqslant 3$. Снова получаем противоречие c (23).

Осталось объяснить, почему для каждого рисунка 16 а)-ж) левый фрагмент слоения всегда можно заменить правым с помошю допустимых преобразований узла $K$ и подходящей деформации диска $F$. Рис. 16 а) и б) соответствуют трюкам Беннекена, которые изначально были определены для кос, при этом вместо нашего слоения использовалось слоение Риба. Однако суть дела здесь та же, что и в [68]. Эти два приема используются в каждой из работ [35]-[40], [51]. Приемы в) и г) были введены в [43]. Приемы д)-ж) являются новыми.

Разберем все случаи по отдельности.

Случай а). Если в слоении $\mathscr{F}$ встретились два соседних одинаково ориентированных внутренних седла, как на рис. 16 a), то это предписывает такой циклический порядок на оси $\ell$ соединенных с ними вершин: $v_{1}, v_{2}, v_{3}, v_{4}, v_{5}, v_{6}$. Рассмотрим, как изменяется сечение $P_{t} \cap F$ при росте $t$. В некоторьй момент $t=t_{1}$ дуги $v_{1} v_{2}$ и $v_{3} v_{4}$ заменяются на $v_{1} v_{4}, v_{2} v_{3}$ в результате перестройки, соответствующей одному из седел на рис. 16 а). Затем вплоть до некоторого момента $t_{2}$ дуга $v_{1} v_{4}$ сохраняется, а при $t=t_{2}$ снова происходит перестройка, в результате которой дуги $v_{1} v_{4}, v_{5} v_{6}$ заменяются на $v_{1} v_{6}, v_{4} v_{5}$. В промежутке $t \in\left(t_{1}, t_{2}\right)$ с сечением $P_{t} \cap F$ могут происходить 
и другие события, но, поскольку дуга $v_{1} v_{4}$ остается при этом нетронутой, их можно разделить на следуюшие две группы: к первой группе отнесем те события, в которых участвуют дуги с концами из $\left(v_{1}, v_{4}\right)$, а ко второй - в которых участвуют дуги с концами из $\left(v_{4}, v_{1}\right)$.

События первой групшы можно свободно переставлять с событиями второй. Поэтому все события из первой групшы можно сдвинуть "в будушее”, т.е. так, чтобы они происходили сразу после $t_{2}$, а события из второй - "в прошлое", т.е. непосредственно перед $t_{1}$. Тогда события

$$
\left(v_{1} v_{2}, v_{3} v_{4}\right) \mapsto\left(v_{1} v_{4}, v_{2} v_{3}\right) \text { и }\left(v_{1} v_{4}, v_{5} v_{6}\right) \mapsto\left(v_{1} v_{6}, v_{4} v_{5}\right)
$$

будут происходить одно за другим, и их можно будет заменить на пару событий

$$
\left(v_{3} v_{4}, v_{5} v_{6}\right) \mapsto\left(v_{3} v_{6}, v_{4} v_{5}\right) \text { и }\left(v_{1} v_{2}, v_{3} v_{6}\right) \mapsto\left(v_{2} v_{3}, v_{1} v_{6}\right)
$$

что отражается на слоении $\mathscr{F}$, как показано на рис. 16 а) справа.

Случай б). Без ограничения общности можно считать $v_{2} \in\left(v_{1}, v_{3}\right)$. Из структуры слоения $\mathscr{F}$ следует, что для некоторых $t_{1}, t_{2}$ мы имеем: $\gamma_{t, v_{1}, v_{2}} \in F$ при $t \in\left(t_{1}, t_{2}\right)$ и $\gamma_{t, v_{2}, v_{3}} \in F$ при $t \in\left(t_{2}, t_{1}\right)$. Это значит, что для достаточно малого $\varepsilon$ мы будем иметь $\gamma_{t, v_{1}^{\prime}, v_{2}^{\prime}} \cap F=\varnothing$ при $t \in\left[t_{1}^{\prime}, t_{2}^{\prime}\right]$ и $\gamma_{t, v_{2}^{\prime}, v_{3}^{\prime}} \cap F=\varnothing$ при $t \in\left[t_{2}^{\prime}, t_{1}^{\prime}\right]$, где $v_{1}^{\prime}=v_{1}+\varepsilon$, $v_{2}^{\prime}=v_{2}-\varepsilon, v_{3}^{\prime}=v_{3}+\varepsilon, t_{1}^{\prime}=t_{1}-\varepsilon, t_{2}^{\prime}=t_{2}+\varepsilon$. Таким образом, мы находимся в ситуации, в которой можно применить обобщенную рокировку и переставить отрезки $\left(v_{1}^{\prime}, v_{2}^{\prime}\right)$ и $\left(v_{2}^{\prime}, v_{3}^{\prime}\right)$ оси $\ell$. Мыприменяем ее не только к узлу $K$, но и к диску $F$, используя те же правила. Слоение на диске $F$ при этом не изменяется. Главным итогом этой обобшенной рокировки является то, что вершины $v_{1}$ и $v_{2}$ становятся соседними, поэтому деформацией диска $F$, затрагивающей лиш поддиск, изображенный на рис. 16 б) слева, мы можем беспрепятственно устранить точки пересечения $v_{1}, v_{2}$ диска $F$ с осью $\ell$. Остальная часть слоения при этом тоже не затрагивается и без ограничения обшности можно считать, что не появляются полюсы (иначе мы могли бы их сразу же удалить, как было сделано в самом начале процесса упрощения диска $F$ ). Отсюда легко видеть, что после такой деформации указанного поддиска слоение в нем вьглядит так, как на рис. 16 б) справа.

Случай в). Этот прием значительно проще. Дуги $\alpha$ и $\beta$ могут беспрепятственно скользить по диску в направлении седла до тех пор, пока не окажутся соседними. При этом узел $K$ претерпевает конечное число перестановок дуг. Когда $\alpha$ и $\beta$ оказываются соседними, их можно заменить на одну дугу с помощью операции дестабилизации.

Случай г). Этот прием аналогичен предыдушему. Дуга $\alpha$ скользит по диску, пока не становится соседней с $\beta$, после чего применяется дестабилизация.

Случай д). Дуга $\alpha$ скользит по диску $F$, пока не достигает седла. Затем мы ее сглаживаем, в результате чего седло, которое теперь стало граничньп, преврашается в “двукратное" граничное седло. Малым шевелением диска $F$ его можно расшепить на пару простых граничных седел.

Случай е). Этот случай аналогичен случаю б). Здесь можно применить обобщенную рокировку к отрезкам $\left(v_{1}-\varepsilon, v_{1}+\varepsilon\right)$ и $\left(v_{1}+\varepsilon, v_{2}-\varepsilon\right)$, первый из которых содержит только вершину $v_{1}$ ( $\varepsilon$ следует выбрать достаточно малым). Вершины $v_{1}, v_{2}$ 
окажутся соседними, и число точек в пересечении $F \cap \ell$ можно будет уменьшить, сократив одну внутреннюю вершину.

Случай ж). Отличие от предыдущего случая состоит в том, что указанные седла одинаково ориентированы. Этот случай подобен случаю а). Диск $F$ можно продеформировать так, чтобы эти седла следовали подряд на $t$-оси. Тогда дуги $\alpha$ и $\beta$ окажутся соседними, и к ним можно будет применить дестабилизацию.

\section{7. Сложность алгоритмов}

Здесь мы кратко обсудим, насколько сложны рассмотренные выше алгоритмы. $\mathrm{C}$ практической точки зрения не имеет большого значения, требует ли алгоритм, скажем, $n^{10}$ или $10^{n}$ операций для выполнения, где $n$-это длина входа. Однако вопрос о сложности алгоритмически трудных задач, к коим несомненно относится задача сравнения узлов, принято обсуждать с точки зрения принадлежности тому или иному сложностному классу, поскольку такая информация подсказывает, насколько хорошего улучшения существуюшего алгоритма можно ожидать. Скажем, если задача оказывается NP-трудной, то, скорее всего, она не имеет полиномиального решения (мы исходим из справедливости гипотезы $\mathrm{N} \neq \mathrm{NP}$ ). Если задача лежит в пересечении классов NP и соNP, то имеет смысл поиск ее полиномиального решения (хотя гипотеза $\mathrm{P}=\mathrm{NP} \cap$ coNP также не доказана).

Из перечисленных вьше алгоритмов только алгоритмы для решения проблемы равенства в группе кос $B_{n}$ являются по-настоящему быстрьми и могут использоваться на практике для кос, длина которых достаточна для любых мыслимых приложений (это не относится к алгоритмам Артина и Гарсайда, которые неоправданно сложны). Вопрос о сложности проблемы равенства в группах $B_{n}$ был поднят Тёрстоном в [31], где он показал, что канонические виды (11) и (12) для косы на $n$ нитях, заданной словом $w$ длины $|w|$, можно найти за $O\left(|w|^{2} \cdot n \log n\right)$ операций.

Кроме того, Тёрстон показал, что если две косы из $B_{n}$ уже представлены в каноническом виде словами $w_{1}, w_{2}$, то найти канонический вид их произведения можно за $O\left(\left|w_{1}\right| \cdot\left|w_{2}\right| \cdot n \log n\right)$ операций.

За счет выбора новых образуюших и соответствуюшего изменения определения канонического вида Бирман, Ко и Ли [64] улучшили оценку сложности алгоритма приведения косы к каноническому виду до $O\left(|w|^{2} \cdot n\right)$.

Алгоритм автора [58] вычисляет $w \cdot(0,1, \ldots, 0,1)$ для действия $(6)$ за $O\left((n+|w|)^{2}\right)$ операций (на RAM-машине), что при больших $n$ сушественно лучше, чем сложность алгоритмов Тёрстона и Бирман-Ко-Ли. Недостатком является то, что вместо какого-либо канонического вида данной косы этот алгоритм выдает набор целых чисел, по которому сама коса не восстанавливается столь же быстро. В совместной работе Б. Виста и автора мы планируем дать полиномиальную оценку времени, необходимого для восстановления косы $b$ по $b \cdot(0,1, \ldots, 0,1)$ и для вычисления $b_{1} b_{2} \cdot(0,1, \ldots, 0,1)$ по данньм $b_{1} \cdot(0,1, \ldots, 0,1)$ и $b_{2} \cdot(0,1, \ldots, 0,1)$.

Алгоритм обрашения слов П. Деорнуа [62] требует $O\left(|w|^{2} \cdot n^{2}\right)$ операций. Другой алгоритм Деорнуа, алгоритм сокращения ручек [63], на практике также работает быстро, но полиномильная оценка его сложности не доказана.

Что касается проблемы сопряженности в группах кос $B_{n}$, то, как мы отмечали выше, вопрос о сложности алгоритма, основанного на нахождении верхушки $\mathscr{S}(b)$, сво- 
дится к оценке размера этой верхушки. Н. Франко и Х. Гонзалез-Менесес [34] показали, что если коса $b \in B_{n}$ задана словом $w$ в $\sigma_{i}$-образующих, то множество $\mathscr{S}(b)$ можно вычислить за $O\left(|w|^{2} n^{4} \cdot|\mathscr{S}(b)|\right)$ операций. Таким образом, если удастся доказать полиномиальную оценку для $|\mathscr{S}(b)|$, то проблему сопряженности в групах кос $B_{n}$ можно будет отнести к классу $\mathrm{P}$.

О сложности алгоритмов для распознавания узлов на сегодняшний день известно немного. В работах [69] и [70] двумя разными методами, используюшими алгоритм Хакена, доказано, что задача распознавания тривиального узла, а также оценки сверху рода узла, лежат в классе NP. Напомним, что принадлежность этих задач классу NP по определению означает, что для каждой пары $(D, g)$, где $D$ - диаграмма некоторого узла, а $g \geqslant 0$ - некоторое целое число, существует сертификат $C$ ("доказательство, что род узла $L_{D}$ не превосходит $g$ "), размер которого полиномиально зависит от сложности записи, кодируюшей $(D, g)$, такой, что некоторьй алгоритм, не зависяший от $D, g$ и $C$, получив на вход $(D, g, C)$, проверяет за полиномиальное время, подтверждает ли сертификат $C$, что род $L_{D}$ не превосходит $g$.

Оба доказательства используют для построения сертификата нормальную поверхность $F$ рода $g$, граница которой совпадает с данным узлом (если он тривиален). Как указьвалось в разделе 3 , такую поверхность можно найти среди фундаментальных. Тогда ее нормальные координаты зависят от сложности $n$ данной диаграммы как $2^{O\left(n^{2}\right)}$, см. [69], а значит, длина записи этих координат растет с $n$ не быстрее, чем полиномиально. Проверить, что данный узел является краем найденной поверхности, и то, что ее эйлерова характеристика равна $2-2 g$, достаточно легко. Трудность, однако, возникает с построением полиномиального алгоритма, проверяющего, что поверхность $F$ связна.

Для преодоления этой трудности в [69] используется тот факт, что поверхность Зейферта наименьшего рода может быть выбрана среди так называемых вершинных решений системы (3), (4), см. [71]. Это такие решения, для которых наибольшее число линейно независимых неравенств (3), ограниченных на пространство решений (4), обращается в равенство. Дж. Хасс, Дж. Лагариас и Н. Пиппенгер включают в сертификат $C$ некоторую вершинную поверхность, и список тех неравенств из (3), проверив линейную независимость которых на пространстве решений (4), можно убедиться, что поверхность действительно вершинная.

И. Эгол, Дж. Хасс и У. Тёрстон [70] показали, как проверить связность поверхности $F$ за полиномиальное время, даже если она не является вершинной. Дело в том, что по заданию нормальной поверхности $F$ в виде решения системы $(3),(4)$ можно описать ее пересечения с ребрами и двумерньми гранями триангуляции объемлющего трехмерного многообразия в следующем виде. Пересечения с ребрами отождествляются с целочисленными точками некоторого интервала $[1, N]$, а пересечения с двумерными гранями задают некоторое отношение на этом интервале: мы будем писать $k \leftrightarrow l$, если точки с номерами $k$ и $l$ соединены отрезком, лежашим в одной двумерной грани триангуляции. И число $N$, и количество пар $(k, l)$, связанных отношением $k \leftrightarrow l$, может быть экспоненциально большим по сравнению со сложностью $n$ данного узла. Однако для записи этих данных достаточно слова длины $O\left(n^{2}\right)$, и такая запись несложно извлекается из нормальных координат поверхности.

Для этого на каждом ребре триангуляии точки пересечения с $F$ упорядочиваются 
естественным образом, после чего каким-либо способом упорядочиваются сами ребpa. Отношение $\leftrightarrow$ задается в виде линейного по $n$ числа отождествлений $[i, j] \leftrightarrow[k, l]$, где $|j-i|=|l-k|$, что означает следуюшее. Пусть $\epsilon=\operatorname{sign}(j-i), \epsilon^{\prime}=\operatorname{sign}(l-k)$. Тогда $[i, j] \leftrightarrow[k, l]$ означает следующий набор соотношений: $i+r \epsilon \leftrightarrow k+r \epsilon^{\prime}, r=$ $0,1, \ldots,|j-i|$.

Итак, задача об определении связности $F$ сводится за полиномиальное время к следуюшей: имеется отрезок $[1, N]$ длины $N=2^{O\left(n^{2}\right)}$ и внутри него $O(n)$ отождествлений вида $[i, j] \leftrightarrow[k, l]$. Требуется определить, сколько классов эквивалентности имеет отношение, порожденное этими отождествлениями. Эгол, Хасс и Тёрстон показали, что ответ на этот вопрос можно найти за полиномиальное по $n$ время.

В работах [69] и [70] доказывается, что задача определения, является ли данное зацепление разводимым или составным, также лежит в NP.

Недавно Эгол предложил доказательство принадлежности классу NP задачи оценки рода узла снизу (не опубликовано). В частности, это означает, что задача распознавания тривиального узла лежит также в coNP, а это дает повод для надежды на существование для нее полиномиального алгоритма.

Заметим, что алгоритм монотонного упрощения, основанный на теореме 6.1 , имеет оценку времени работы $2^{O(n \log n)}$, но не дает нового доказательства принадлежности задачи классу NP. Для того, чтобы получить такое доказательство, нужно найти полиномиальную оценку сверху для длины упрощающей последовательности. Единственная оценка, которая на сегодня известна автору, вытекает из оценки на общее число прямоугольных диаграмм сложности $n$, см. предложение 2.1. По-видимому, эту оценку можно существенно улучшить.

Что касается задачи сравнения произвольных узлов и зацеплений, о ее сложности ничего не известно. В частности, для времени работы алгоритма Матвеева не известно никакой оценки сверху, выраженной в элементарных функциях.

Для алгоритма Бирман-Хирша распознавания тривиального узла оценку времени работы выписать можно, но она будет заведомо не лучше, чем для алгоритма Хакена.

\section{8. Некоторые новые алгоритмические задачи теории узлов}

В этом разделе сформулировано несколько новых задач, которые естественно возникли при изучении автором вложений узлов в объединения полуплоскостей.

8.1. Флайпы прямоугольных диаграмм. Здесь мы введем единообразно описьваемое семейство преобразований прямоугольных диаграмм, содержащее как частные случаи все рокировки, (де)стабилизации, а также отражения относительно диагоналей. Мы назьваем эти преобразования флайпами. Нам будет удобнее пользоваться языком геометрических прямоугольных диаграмм.

Пусть $R$-некоторая геометрическая прямоугольная диаграмма, $a, b$-положительные числа такие, что все вершины диаграммы $R$ лежат в

$\left(\mathbb{R}^{2} \backslash(0, a+b) \times(0, a+b)\right) \cup(0, a) \times(0, b) \cup\{(a+t, t) \mid t \in(0, b)\} \cup\{(t, b+t) \mid t \in(0, a)\}$,

а также ни одна точка на отрезках $\{(a+t, t) \mid t \in(0, b)\}$ и $\{(t, b+t) \mid t \in(0, a)\}$, не являюшаяся вершиной диаграммы $R$, не лежит на пересечении двух прямых, содержаших ребра диаграммы $R$. 
Построим новую прямоугольную диаграмму $R^{\prime}$, заменив каждую вершину $(x, y) \in$ $(0, a) \times(0, b)$ на $(y+a, x+b)$. На отрезках $\{(a+t, t) \mid t \in(0, b)\}$ и $\{(t, b+t) \mid$ $t \in(0, a)\}$ вершины удаляются или добавляются, исходя из того, чтобы в результате получился набор вершин некоторой прямоугольной диаграммы. Это можно сделать единственньм способом. См. пример на рис. 19.
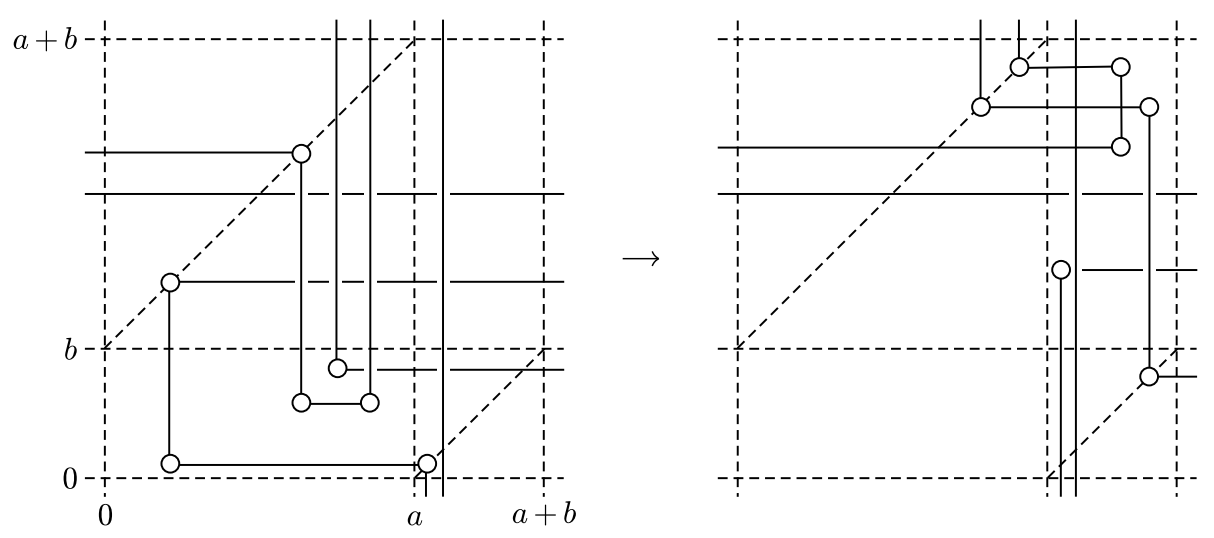

Рис. 19. Пример фолайпа

Преобразование $R \mapsto R^{\prime}$, а также любое, сопряженное ему с помошњю поворота на $\pi / 2$ или $\pi$, назовем флайпом. По нашему определению преобразование, обратное к флайпу, также является флайпом. Мы выбрали такое название для описанной только что операции $R \mapsto R^{\prime}$ потому, что, если рассматривать $R$ как обычную плоскую диаграмму, в некоторых случаях действие нашей операции совпадает с операцией, называемой флайпом в литературе. Однако в общем случае флайп в нашем смысле может давать совершенно другие преобразования. Например, если вся диаграмма $R$ находится внутри прямоугольника $(0, a) \times(0, b)$, то флайп $R \mapsto R^{\prime}$ сводится к отражению относительно прямой $x=y$ и сдвигу, который для нас несушественен. На рис. 20 продемонстрировано, что стабилизация и рокировка также являются частными случаями фолайпов.

ПРЕДЛОЖЕНИЕ 8.1. Определенная выше операция флайпа $R \mapsto R^{\prime}$ всегда сохраняет изотопический тип зачепления: $L_{R} \sim L_{R^{\prime}}$.

ЗАДАчА 1. Изучить возможности флайпов в обшем случае. Всегда ли можно уменьшить сложность диаграммы тривиального узла, применив всего один флайп? Всегда ли фолайпами можно перейти от одной прямоугольной диаграммы к другой, не увеличивая сложность, если эти диаграммы задают изотопные зацепления?

Эти вопросы кажутся автору интересными сами по себе. Кроме того, положительньй ответ на первьй вопрос мог бы позволить построить полиномиальньй алгоритм для распознавания тривиального узла, а положительный ответ на второй вопрос мог бы позволить установить принадлежность задачи сравнения зацеплений классу NP.

8.2. Алгоритм Хакена и каноническая триангуляция. По любому комбинаторному описанию зацепления можно так или иначе построить триангуляцию его 

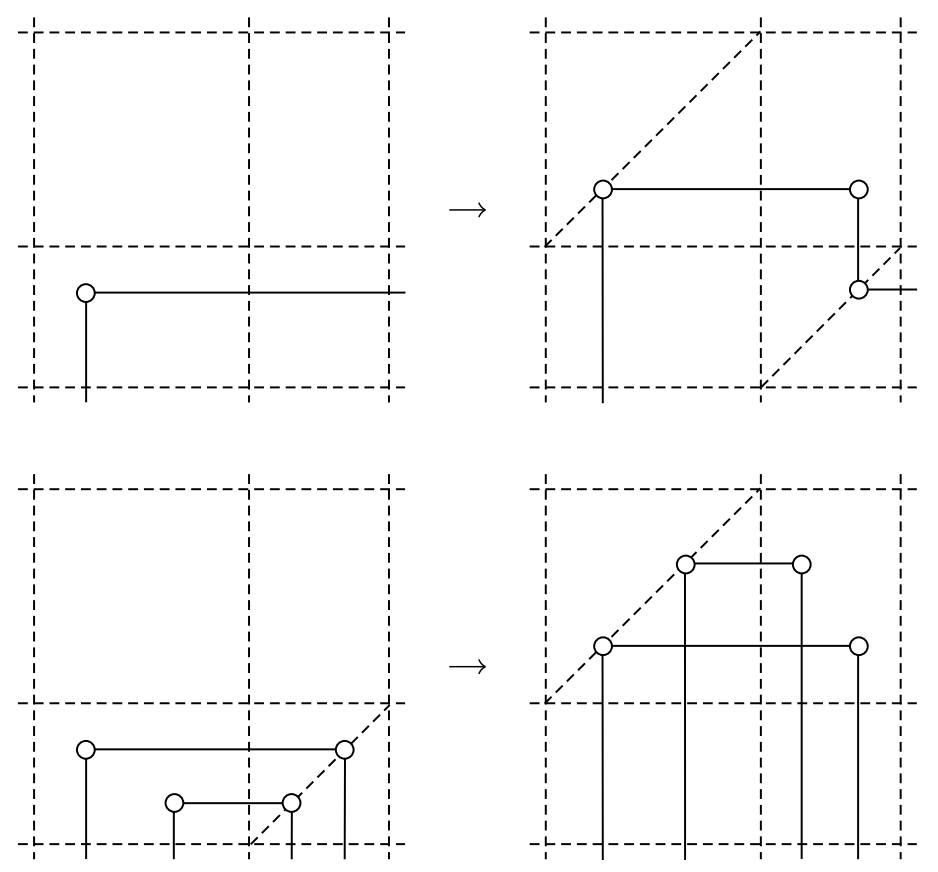

Рис. 20. Стабилизация и рокировка как частные случаи флайпов

дополнительного пространства. В случае прямоугольных диаграмм имеется канонический способ указать триангуляцию $T$ сферы $S^{3}$ такую, что данное зацепление будет реализовано подмножеством одномерного скелета триангуляци $T$. Это делается следуюшим образом.

Пусть $R$-прямоугольная диаграмма сложности $n$. Представим $S^{3}$ как джойн двух окружностией, $S^{3}=S^{1} * S^{1}$. На каждой из окружностей отметим $n$ точек и занумеруем их в соответствии с ориентацией окружности. Пусть $A_{1}, \ldots, A_{n}$ - точки на первой окружности, $B_{1}, \ldots, B_{n}-$ на второй. Для каждой вершины $(i, j) \in R$ нашей прямоугольной диаграммы соединим точку $A_{i}$ с $B_{j}$ отрезком $A_{i} * B_{j} \subset S^{1} * S^{1}$. Получим зацепление $L_{R}$. Оно будет подмножеством одномерного остова триангуляции сферы $S^{3}, 3$-симплексами которой являются $\left[A_{i}, A_{i+1}\right] *\left[B_{j}, B_{j+1}\right] \subset S^{1} * S^{1}, i, j \in 1,2, \ldots, n$, где индексы рассматриваются по модулю $n$, а $[C, D]$ обозначает дугу окружности $S^{1}$ между точками $C$ и $D$. (Формально говоря, мы должны были потребовать $n \geqslant 3$, чтобы получить настоящую комбинаторную триангулящию.) Обозначим эту триангуляцию через $T_{n}$. Она зависит только от сложности $n$ данной диаграммы $R$.

ЗАДАчА 2. Изучить возможности эффективизации метода Хакена при использовании триангулящий $T_{n}$. Для этой триангуляции описать явно все фундаментальные сферы.

Такая постановка вызвана тем, что в ряде конкретных примеров удается показать, что эйлерова характеристика поверхности, нормальной по отношению к $T_{n}$ и не пересекающей $L_{R}$, линейно выражается через нормальные координаты с неположительными коэффищиентами. Это позволяет сразу сделать вывод о несушествовании нор- 
мальных сфер в дополнении к $L_{R}$. Эта ситуация имеет место, например, для всех "жестких" зацеплений, т.е. не допускаюших ни одной рокировки и дестабилизации.

Заметим, что умение быстро находить фундаментальные сферы в дополнении к зацеплению означает существование быстрого алгоритма для распознавания разводимого зацепления. Последнее в свою очередь влечет наличие быстрого алгоритма для распознавания тривиального узла.

Действительно, пусть $K$-некоторьй узел. Рассмотрим зацепление, получающееся заменой $K$ на две его параллельные копии, имеюшие коэффициент зацепления нуль. Легко показать, что $L$ разводимо тогда и только тогда, когда узел $K$ тривиален.

Если каким-то образом удастся описать все фундаментальные сферы для триангулящии $T_{n}$, то для построения алгоритма распознавания тривиального узла достаточно будет научиться эффективно отбирать среди этих сфер те, которые не пересекают данное семейство ребер.

8.3. Сведение классификации зацеплений к проблеме равенства в конечно определенной полугруппе. Пусть $Y_{3}$ - полугруппа, заданная множеством образующих $\left\{a_{i}, b_{i}, c_{i}, d_{i}\right\}_{i \in \mathbb{Z}_{3}}$ и соотношениями

$$
\begin{gathered}
a_{i}=a_{i+1} d_{i-1}, \quad b_{i}=a_{i-1} c_{i+1}, \quad c_{i}=b_{i-1} c_{i+1}, \quad d_{i}=a_{i+1} c_{i-1}, \\
d_{1} d_{2} d_{3}=1, \quad b_{i} d_{i}=d_{i} b_{i}=1, \\
x_{i} y_{i}=y_{i} x_{i},
\end{gathered}
$$

где $x_{i} \in\left\{a_{i-1} b_{i-1}, d_{i-1} c_{i-1}, b_{i+1} d_{i-1} d_{i+1} b_{i-1}\right\}, y_{i} \in\left\{a_{i}, b_{i}, c_{i}, b_{i-1} d_{i} d_{i-1}\right\}, i \in \mathbb{Z}_{3}$.

Пусть $Y_{4}$ - полугруппа, заданная множеством образуюших $\left\{a_{i}, b_{i}, c_{i}, d_{i}\right\}_{i \in \mathbb{Z}_{4}}$ и соотношениями

$$
\begin{gathered}
a_{i} d_{i+1}=a_{i+1} b_{i}, \quad d_{i} c_{i+1}=b_{i+1} c_{i}, \quad a_{i} d_{i}=a_{i+1} b_{i+1}, \quad b_{i} c_{i}=d_{i+1} c_{i+1}, \\
d_{1} d_{2} d_{3} d_{4}=1, \quad a_{i} b_{i} c_{i} d_{i}=1, \quad b_{i} d_{i}=d_{i} b_{i}=1, \\
x_{i} y_{i+2}=y_{i+2} x_{i},
\end{gathered}
$$

где $x_{i} \in\left\{a_{i}, b_{i}, c_{i}, d_{i}\right\}, y_{i} \in\left\{a_{i}, b_{i}, c_{i}, d_{i}, b_{i-1} a_{i} d_{i-1}, b_{i-1} b_{i} d_{i-1}, b_{i-1} c_{i} d_{i-1}\right\}, i \in \mathbb{Z}_{4}$.

При $n \geqslant 5$ пусть $Y_{n}$ - полугруппа, заданная множеством образующих $\left\{a_{i}, b_{i}\right.$, $\left.c_{i}, d_{i}\right\}_{i \in \mathbb{Z}_{n}}$ и соотношениями

$$
\begin{gathered}
a_{i} d_{i+1}=a_{i+1} b_{i}, \quad d_{i} c_{i+1}=b_{i+1} c_{i}, \quad a_{i} d_{i}=a_{i+1} b_{i+1}, \quad b_{i} c_{i}=d_{i+1} c_{i+1}, \\
d_{1} d_{2} \cdots d_{n}=1, \quad a_{i} b_{i} c_{i} d_{i}=1, \quad b_{i} d_{i}=d_{i} b_{i}=1, \\
x_{i} y_{j}=y_{j} x_{i}
\end{gathered}
$$

для всех $i, j \in \mathbb{Z}_{n}$ таких, что $i-j \notin\{-1,0,1\}$ и $x_{i} \in\left\{a_{i}, b_{i}, c_{i}, d_{i}\right\}, y_{j} \in\left\{a_{j}, b_{j}, c_{j}, d_{j}\right\}$.

Наконец, при $n \geqslant 5$ пусть $Z_{n}$ - полугруппа, заданная множеством образуюших $\left\{a_{i}, c_{i}\right\}_{i \in \mathbb{Z}_{2 n}}$ и соотношениями

$$
\begin{gathered}
a_{i \pm 1} x_{i} c_{i \pm 1}=x_{i}, \quad a_{i} c_{i \pm 1} a_{i \pm 1} c_{i}=1, \\
a_{1} c_{0} a_{3} c_{2} \cdots a_{2 n-1} c_{2 n-2}=1, \quad a_{2} c_{1} a_{4} c_{3} \cdots a_{2 n} c_{2 n-1}=1, \\
x_{i} y_{j}=y_{j} x_{i},
\end{gathered}
$$

где $i, j \in \mathbb{Z}_{2 n}, x_{i} \in\left\{a_{i}, c_{i}\right\}, y_{j} \in\left\{a_{j}, c_{j}\right\}, i-j \neq-1,0,1 \in \mathbb{Z}_{2 n}$. 
В работах [72]-[75] неориентированные зацепления реализованы как центральные элементы каждой из полугруп $Y_{n}, n \geqslant 3$. Конструкция основана на рассмотрении так назьваемых $n$-страничных зацеплений, которые напоминают книжные зацепления. Различие в том, что число страниц, которые могут содержать дуги зацепления, фиксировано, но в каждой из страниц может быть любое количество дуг. Такие зацепления несложным образом кодируются словами в алфавите $\left\{a_{i}, b_{i}, c_{i}, d_{i}\right\}_{i \in \mathbb{Z}_{n}}$, а указанные вьше соотношения порождают отношение изотопности.

Подобным образом в работе [76] ориентированные зацепления реализованы как центральные элементы каждой из полугруп $Z_{n}, n \geqslant 5$.

ЗАДАчА 3 . Решить проблему равенства в полугруппах $Y_{n}$ и $Z_{n}$ алгебраическими средствами.

Решение этой задачи означало бы новое решение проблемы алгоритмической классификации зацеплений. Заметим, что, хотя зацеплениям соответствуют только центральные элементы полугруппы $Y_{n}$ или $Z_{n}$, существование алгоритма сравнения многообразий Хакена с узором на крае влечет разрешимость проблемы равенства во всей полугрупе, а не только в центре. Таким образом, мы уже знаем, что эта задача разрешима, вопрос лишш в том, можно ли разумным образом изложить ее решение в алгебраических терминах, не прибегая к топологическим аргументам.

8.4. Вложение группы кос в конечно определенные группы. В работах [72]-[75] были также описаны групш $D_{n}$ обратимых элементов каждой из полугруп $Y_{n}$. Групша $D_{n}$ задается образуюшими $\left\{d_{i}\right\}_{i \in \mathbb{Z}_{n}}$ и теми из соотношений в задании $Y_{n}$, которые содержат только буквы $d_{i}$.

А именно, группа $D_{3}$ задается соотношениями

$$
\begin{gathered}
d_{1} d_{2} d_{3}=1, \\
d_{i} u_{i}=u_{i} d_{i}, \quad u_{i} u_{i+1}=u_{i+1} u_{i},
\end{gathered}
$$

где $u_{i}=d_{i+1}^{-1} d_{i-1} d_{i+1} d_{i-1}^{-1}, i \in \mathbb{Z}_{3}$.

Группа $\mathrm{D}_{4}$ задается соотношениями

$$
\begin{gathered}
d_{1} d_{2} d_{3} d_{4}=1, \\
d_{i} d_{i+2}=d_{i+2} d_{i}, \quad d_{i+2}\left(d_{i-1}^{-1} d_{i} d_{i-1}\right)=\left(d_{i-1}^{-1} d_{i} d_{i-1}\right) d_{i+2},
\end{gathered}
$$

где $i \in \mathbb{Z}_{4}$.

При $n \geqslant 5$ групша $D_{n}$ задается соотношениями

$$
\begin{gathered}
d_{1} d_{2} \cdots d_{n}=1 \\
d_{i} d_{j}=d_{j} d_{i}, \text { если } i-j \neq \pm 1
\end{gathered}
$$

где $i, j \in \mathbb{Z}_{n}$

В [72]-[75] был доказан следуюший результат.

ТЕОРема 8.1. Для любого $n \geqslant 3$ коммутант группь $D_{n}$ изоморфен группе кос $B_{\infty}$ на бесконечном числе нитей. 
Под группй $B_{\infty}$ подразумевается прямой предел последовательности групп $B_{1} \subset$ $B_{2} \subset B_{3} \subset \cdots$. Изоморфизм $B_{\infty} \cong\left[D_{n}, D_{n}\right]$ во всех случаях можно описать явно в терминах образующих.

ЗАДАчА 4 . Исследовать групшы $D_{n}$, исходя из указанных вьше заданий образующими и соотношениями. Найти для этих групп эффективное решение проблемы сопряженности, независимое от решения аналогичной задачи для групп кос.

Групшы $D_{n}$ очень близки по структуре к групам кос, однако задаются образуюшими и соотношениями совершенно по-другому. Не исключено, что специфика этих соотношений позволит решить проблему сопряженности эффективнее, хотя и может случиться, что с введением "более простых" соотношений задача лишш усложнится. Во всяком случае, автору кажется, что новые соотношения заслуживают того, чтобы взглянуть на них с чисто алгебраической точки зрения.

\section{СПИСОК ЛИТЕРАТУРЫ}

[1] T.P. Kirkman. The enumeration, description and construction of knots of fewer that ten crossings // Trans. Roy. Soc. Edinburgh. 1885. V. 32. P. 281-309.

[2] P. G. Tait. On knots // Trans. Roy. Soc. Edinburgh. 1877. V. 28. P. 145-190.

[3] J.W. Alexander, G. B. Briggs. On types of knotted curved // Ann. of Math. 1926/27. V. 28. P. $562-586$.

[4] K. Reidemeister. Knotentheorie. Berlin: Springer-Verlag, 1932. (Ergeb. Math. Grenzgeb. V. 1. № 1.)

[5] J. W. Alexander. A lemma on systems of knotted curves // Proc. Natl. Acad. Sci. USA. 1923. V. 9. P. 93-95.

[6] A. A. Markoff. Über die freie Äquivalenz der geschlossenen Zöpfe // Матем. сб. 1936. T. 1 (43). № 1. C. $73-78$.

[7] E. Artin. Theorie der Zöpfe // Abh. Math. Sem. Univ. Hamburg. 1925. V. 4. P. 47-72.

[8] E. Artin. Theory of braids // Ann. of Math. (2). 1947. V. 48. № 1. P. 101-126.

[9] W. Haken. Theorie der Normalflächen. Ein Isotopiekriterium für der Kreisknoten // Acta Math. 1961. V. 105. № 3-4. P. 245-375.

[10] H. Kneser. Geschlossene Flächen in dreidimensionalen Mannigfaltigkeiten // Jahresber. Deutsch. Math.-Verein. 1929. V. 38. P. 248-260.

[11] H. Schubert. Bestimmung der Primfactorzerlegung von Verkettungen // Math. Z. 1961. V. 76. Р. 116-148. (Рус. пер.: Х. Шуберт. Алгоритм для разложения зацеплений на простые слагаемые // Математика: Сб. перев. 1966. Т. 10. № 4. С. 45-78.)

[12] H. Schubert. Die eindeutige Zerlegbarkeit eines Knotens in Primknoten // S.-B. Heidelberger Akad. Wiss. Math.-Nat. Kl. 1949. V. 1949. № 3. P. 57-104.

[13] Y. Hashizume. On the uniqueness of the decomposition of a link // Osaka Math. J. 1958. V. 10. P. 283-300; 1959. V. 11. P. 249.

[14] W. Haken. Ein Verfahren zur Aufspaltung einer 3-Mannigfaltigkeit in irreduzible 3-Mannigfaltigkeiten // Math. Z. 1961. V. 76. P. 427-467.

[15] W. Haken. Über das Homöomorphieproblem der 3-Mannigfaltigkeiten. I // Math. Z. 1962. V. 80. P. $89-120$.

[16] F. Waldhausen. On irreducible 3-manifolds which are sufficiently large // Ann. of Math. (2). 1968. V. 87. P. 56-88.

[17] K. Johannson. Homotopy Equivalences of 3-Manifolds with Boundaries. Berlin: SpringerVerlag, 1979. (Lecture Notes in Math. V. 761.)

[18] W. Jaco, P. Shalen. Seifert fibered spaces in 3-manifolds // Mem. Amer. Math. Soc. 1979. V. 21. № 220 .

[19] G. Hemion. On the classification of homeomorphisms of 2-manifolds and the classification of 3-manifolds // Acta Math. 1979. V. 142. №1-2. P. 123-155. 
[20] F. Waldhausen. Recent results on sufficiently large 3-manifolds // Algebraic and Geometric Topology. Part 2. Providence, RI: Amer. Math. Soc., 1978. P. 21-38. (Proc. Sympos. Pure Math. V. 32.)

[21] K. Johannson. Classification problems in low-dimensional topology // Geometric and Algebraic Topology.. Warsaw: PWN, 1986. P. 37-59. (Banach Center Publ. V. 18.)

[22] G. Hemion. The Classification of Knots and 3-Dimensional Spaces. New York: Clarendon Press, Oxford Univ. Press, 1992.

[23] S. V. Matveev. On the recognition problem for Haken 3-manifolds // Proceedings of the Workshop on Differential Geometry and Topology (Palermo, June 3-9, 1996) / ed. R. Grimaldi et al. Palermo: Circolo Matematico di Palermo, 1997. P. 131-148. (Suppl. Rend. Circ. Mat. Palermo. V. 49.)

[24] W. Thurston. On the geometry and dynamics of diffeomorphisms of surfaces // Bull. Amer. Math. Soc. (N.S.). 1988. V. 19. № 2. P. 417-431.

[25] M. Bestvina, M. Handel. Train-tracks for surface homeomorphisms // Topology. 1995. V. 34. №1. P. 109-140.

[26] S. V. Matveev. Algorithmic Topology and Classification of 3-Manifolds. Berlin: SpringerVerlag, 2003. (Algorithms Comput. Math. V. 9.)

[27] W. P. Thurston. Three dimensional manifolds, Kleinian groups and hyperbolic geometry // Bull. Amer. Math. Soc. (N.S.). 1982. V. 6. P. 357-379.

[28] M. Kapovich. Hyperbolic Manifolds and Discrete Groups. Boston, MA: Birkhäuser, 2001. (Progr. Math. V. 183.)

[29] Г. С. Маканин. Проблема сопряженности в группе кос // Докл. АН СССР. 1968. Т. 182. № 3. C. 495-496.

[30] F. A. Garside. The braid group and other groups // Quart. J. Math. Oxford Ser. (2). 1969. V. 20. № 78. P. 235-254.

[31] D. B. A. Epstein, J.W. Cannon, D. F. Holt, S. V.F. Levy, M.S. Patterson, W. P. Thurston. Word Processing in Groups. Boston: Jones and Bartlett, 1992.

[32] E. A. El-Rifai, H. R. Morton. Algorithms for positive braids // Quart. J. Math. Oxford Ser. (2). 1994. V. 45. № 180. P. 479-497.

[33] J. Birman, K. H. Ko, S. J. Lee. The infimum, supremum, and geodesic length of a braid conjugacy class // Adv. Math. 2001. V. 164. № 1. P. 41-56.

[34] N. Franco, J. González-Meneses. Conjugacy problem for braid groups and Garside groups // J. Algebra. 2003. V. 266. № 1. P. 112-132.

[35] J. Birman, W. Menasco. Studying links via closed braids. I: A finiteness theorem // Pacific J. Math. 1992. V. 154. №1. P. 17-36.

[36] J. Birman, W. Menasco. Studying links via closed braids. II: On a theorem of Bennequin // Topology Appl. 1991. V. 40. № 1. P. 71-82.

[37] J. Birman, W. Menasco. Studying links via closed braids. III: Classifying links which are closed 3-braids // Pacific J. Math. 1993. V. 161. №1. P. 25-113.

[38] J. Birman, W. Menasco. Studying links via closed braids. IV: Composite links and split links // Invent. Math. 1990. V. 102. № 1. P. 115-139.

[39] J. Birman, W. Menasco. Studying links via closed braids. V: The unlink // Trans. Amer. Math. Soc. 1992. V. 329. № 2. P. 585-606.

[40] J. Birman, W. Menasco. Studying links via closed braids. VI: A nonfiniteness theorem // Pacific J. Math. 1992. V. 156. № 2. P. 265-285.

[41] J. Birman, W. Menasco. Stabilization in the braid groups // http://arxiv.org/abs/ math.gt/0310279.

[42] J. Birman, M. Hirsch. A new algorithm for recognizing the unknot // Geom. Topol. 1998. V. 2. № 9. P. 175-220.

[43] I. A. Dynnikov. Arc-presentations of links. Monotonic simplification // arxiv.org/abs/ math.gt/0208153.

[44] J. H. Conway. An enumeration of knots and links, and some of their algebraic properties // Computational Problems in Abstract Algebra (Proc. Conf., Oxford, 1967). Oxford: Pergamon, 1970. P. 329-358. 
[45] C. H. Dowker, M.B. Thistlethwaite. Classification of knot projections // Topol. Appl. 1983. V. 16. №1. P. 19-31.

[46] J. Hoste, M. Thistlethwaite, J. Weeks. The first 1,701,936 knots // Math. Intelligencer. 1998. V. 20. № 4. P. 33-48.

[47] F. Jaeger, D. L. Vertigan, D. J. A. Welsh. On the computational complexity of the Jones and Tutte polynomials // Math. Proc. Cambridge Philos. Soc. 1990. V. 108. № 1. P. $35-53$.

[48] R.H. Crowell, R.H. Fox. Introduction to Knot Theory. Boston: Ginn, 1963. (Рус. пер.: Р. Кроуэлл, Р. Фокс. Введение в теорию узлов. М.: Мир, 1967.)

[49] G. Burde, H. Zieschang. Knots. Berlin: de Gruyter, 1985. (de Gruyter Stud. Math. V. 5.)

[50] J. Birman. Braids, Links, and Mapping Class Groups. Princeton / Tokyo: Princeton Univ. Press / Univ. of Tokyo Press, 1974. (Ann. of Math. Stud. V. 82.)

[51] P. Cromwell. Embedding knots and links in an open book. I: Basic properties // Topology Appl. 1995. V. 64. P. 37-58.

[52] P. Vogel. Representation of links by braids: A new algorithm // Comment. Math. Helv. 1990. T. 65. № 1. C. 104-113.

[53] W. D. Neumann, G. A. Swarup. Canonical decompositions of 3-manifolds // Geom. Topol. 1997. V. 1. P. 21-40.

[54] J. Hass. Algorithms for recognizing knots and 3-manifolds // Chaos Solitons Fractals. 1998. V. 9. № 4-5. P. 569-581.

[55] F. Bohnenblust. The algebraical braid group // Ann. of Math. (2). 1947. V. 48. P. $127-136$.

[56] W.-L. Chow. On the algebraical braid group // Ann. of Math. (2). 1948. V. 49. № 3. P. 654-658.

[57] P. Dehornoy, I. Dynnikov, D. Rolfsen, B. Wiest. Why are Braids Orderable? Paris: Soc. Math. France, 2002. (Panor. Synthèses. V. 14.)

[58] И.А. Дынников. Об одном отображении Янга-Бакстера и упорядочении Деорнуа // УМH. 2002. Т. 57. №3. С. 151-152.

[59] P. Dehornoy. Braids and Self-Distributivity. Basel: Birkhäuser, 2000. (Progr. Math. V. 192.)

[60] А. В. Малютин. Быстрые алгоритмы распознавания и сравнения кос // Зап. науч. семин. ПОМИ. 2001. Т. 279. С. 200-215.

[61] С. И. Адян. О фрагментах слова $\Delta$ в группе кос // Матем. заметки. 1984. Т. 36. № 1. C. $25-34$.

[62] P. Dehornoy. Groups with a complemented presentation // J. Pure Appl. Algebra. 1997. V. 116. № 1-3. P. 115-137.

[63] P. Dehornoy. A fast method for comparing braids // Adv. Math. 1997. V. 125. №2. P. $200-235$.

[64] J. Birman, K. H. Ko, S. J. Lee. A new approach to the word and conjugacy problems in the braid groups // Adv. Math. 1998. V. 139. № 2. P. 322-353.

[65] J.S. Birman, P. Boldi, M. Rampichini, S. Vigna. Towards an implementation of the B-H algorithm for recognizing the unknot // J. Knot Theory Ramifications. 2002. V. 11. № 4. P. $601-645$.

[66] L. Goeritz. Bemerkungen zur knotentheorie // Abh. Math. Sem. Univ. Hamburg. 1934. V. 10. P. 201-210.

[67] H. R. Morton. An irreducible 4-string braid with unknotted closure // Math. Proc. Cambridge Philos. Soc. 1983. V. 93. № 2. P. 259-261.

[68] D. Bennequin. Entrelacements et équations de Pfaff // Astérisque. 1983. V. 107-108. Р. 87-161. (Рус. пер.: Д. Беннекен. Зацепления и уравнения Пфаффа // УМН. 1989. Т. 44. № 3. C. $3-53$.)

[69] J. Hass, J.C. Lagarias, N. Pippenger. The computational complexity of knot and link problems // J. ACM. 1999. V. 46. № 2. P. 185-211.

[70] I. Agol, J. Hass, W. Thurston. The computational complexity of knot genus and spanning area // http://arxiv.org/abs/math.gt/0205057. 
[71] W. Jaco, J.L. Tollefson. Algorithms for the complete decomposition of a closed 3-manifold // Illinois J. Math. 1995. V. 39. № 3. P. 358-406.

[72] И.А. Дынников. Трехстраничные представления узлов. Кодирование и локальные движения // Функц. анализ и его прил. 1999. Т. 33. № 4. С. 25-37.

[73] И.А. Дынников. Трехстраничные представления узлов. Универсальная полугруппа // Функц. анализ и его прил. 2000. Т. 34. № 1. С. 29-40.

[74] И. А. Дынников. Конечно определенные группы и полугруппы в теории узлов // Труды МИАН. 2000. Т. 231. С. 231-248.

[75] I. A. Dynnikov. A new way to represent links. One-dimensional formalism and untangling technology // Acta Appl. Math. 2001. V. 69. № 3. P. 243-283.

[76] I. A. Dynnikov. Finitely presented semigroups in knot theory. Oriented case // Geometry, Topology and Mathematical Physics: S. P. Novikov's Seminar 2001-2003 / ed. V. M. Buchstaber, I. M. Krichever. Amer. Math. Soc. Tranls. Ser. 2, 2004 (to appear).

Московский государственньй

университет им. М. В. Ломоносова,

Laboratoire international franco-russe de mathématiques

et interactions en informatique et en physique théorique

E-mail: dynnikov@mech.math.msu.su
Поступила в редакцию

07.10 .2003 